\title{
Distinct VIP interneurons in the cingulate cortex encode anxiogenic and social stimuli
}

Connor Johnson ${ }^{1 \#}$, Lisa N. Kretsge ${ }^{2,3 \#}$, William W. Yen"1\#, Balaji Sriram ${ }^{4}$, Jessica C. Jimenez ${ }^{5}$, Tushare J. Jinadasa ${ }^{1}$, Alexandra O'Connor ${ }^{6}$, Ruichen Sky Liu ${ }^{7}$, Thanh P. H. Nguyen ${ }^{6}$, Eun Seon Cho $^{1}$, Erelle Fuchs ${ }^{1}$, Eli D. Spevack ${ }^{1}$, Berta Escude Velasco ${ }^{1}$, Frances S. Hausmann ${ }^{1}$, Alberto Cruz-Martín ${ }^{1,3,9,10,11, *}$

${ }^{1}$ Department of Biology, Boston University, Boston, Massachusetts, United States of America, ${ }^{2}$ The Graduate Program for Neuroscience, Boston University, Boston, Massachusetts, United States of America, ${ }^{3}$ Neurophotonics Center, Boston University, Boston, Massachusetts, United States of America, ${ }^{4}$ Research and Early Development, Biogen, Cambridge, Massachusetts, United States of America, ${ }^{5}$ Department of Neurology, University of California, San Francisco, San Francisco, California, USA, ${ }^{6}$ Department of Biomedical Engineering, Boston University, Boston, Massachusetts, ${ }^{7} \mathrm{MS}$ in Statistical Practice, Boston University, Boston, Massachusetts, United States of America, United States of America, ${ }^{8}$ Department of Computer Science, Boston University, Boston, Massachusetts, United States of America, ${ }^{9}$ Molecular Biology, Cell Biology and Biochemistry Program, Boston University, Boston, Massachusetts, United States of America, ${ }^{10}$ Department Pharmacology and Experimental Therapeutics, Boston University, Boston, Massachusetts, United States of America, ${ }^{11}$ Center for Systems Neuroscience, Boston University, Boston, Massachusetts, United States of America

\#Co-first author

*Corresponding author

Corresponding author contact information:

Name: Alberto Cruz-Martín

Postal address: Boston University, 24 Cummington Mall, Room 426, Boston, MA 02215

Tel. \#: 617-358-3291

Fax \#: 617-353-8484

E-mail address: acmartin@bu.edu

\section{ABSTRACT}

A hallmark of higher-order cortical regions is their functional heterogeneity, but it is not well understood how these areas encode such diverse information. The anterior cingulate cortex (ACC), for example, is important in both emotional regulation and social cognition. Previous work shows activation of the ACC to anxiety-related and social stimuli, but it is unknown how subpopulations or microcircuits within the ACC simultaneously encode these distinct stimuli. One type of inhibitory interneuron, which is positive for vasoactive intestinal peptide (VIP), is known to alter the activity 
40 of many cells in local cortical microcircuits, but it is unknown whether the activity of VIP cells in

41 the ACC (VIP ${ }^{\mathrm{ACC}}$ ) encodes anxiety-related or social information. Using in vivo calcium imaging

42 and miniscopes in freely behaving mice to monitor VIPACC activity, we identified distinct, non-

43 overlapping subpopulations of VIP ${ }^{\mathrm{ACC}}$ that preferentially activated to either anxiogenic, anxiolytic,

44 social, or non-social stimuli. We determined that stimulus-selective cells encode the animal's

45 behavioral states and VIP interneuron clusters may co-activate, improving this encoding. Finally,

46 we used trans-synaptic tracing to show that VIP ${ }^{A C C}$ receive widespread inputs from regions

47 implicated in emotional regulation and social cognition. These findings demonstrate not only that

48 the ACC is not homogeneous in its function, but also that there is marked functional heterogeneity

49 even within disinhibitory interneuron populations. This work contributes to our understanding of

50 how the cortex encodes information across diverse contexts and provides insight into the complexity

51 of neural processes involved in anxiety and social behavior.

INTRODUCTION

55 Cortical subregions are often implicated in a variety of behavioral functions, but it is not well

56 understood how these areas encode such diverse information. The anterior cingulate cortex (ACC)

57 is necessary for emotional processing and social cognition, but how it encodes stimuli relevant to

58 both processes is unknown (1-6). In humans, ACC activity increases when healthy subjects

59 perform social tasks and is higher in anxiety disorder patients who demonstrate increased symptom

60 severity (7). In rodents and non-human primates, ACC inhibition via chemical lesions or genetic

61 manipulations impairs social behaviors (8-10). In addition, stimulating activity or silencing a

62 cytoskeletal protein in the ACC alters anxiety-like behaviors $(11,12)$. Recent studies in rodents 
63 have provided insight into the ACC's importance in emotional and social behaviors by monitoring

64 either single unit or bulk activity of this region $(6,8,11,13-16)$. However, these data do not parse

65 out the roles of different neural subtypes or microcircuits within the ACC. It remains unknown

66 how neuronal representations of diverse stimuli are embedded within ACC subcircuits.

Inhibitory neurons that are positive for vasoactive intestinal peptide (VIP) inhibit other

68 inhibitory cells, thereby driving excitatory pyramidal cell (Pyr) activity (17-20). VIP interneurons,

69 therefore, are in a unique position to modulate local activity $(21,22)$. They also receive long-range

70 inputs from other brain regions, which may allow them to coordinate the activity of the ACC with

71 other brain regions to respond to diverse stimuli $(18,20,23)$. In the hippocampus, VIP interneurons

72 form functional clusters that are differently modulated by behavioral states, but this has not been

73 studied in higher-order cortical regions like the ACC (24). Acetylcholine and serotonin can

74 modulate functionally distinct groups of VIP interneurons and may differentially engage them

75 during diverse behaviors (25-27). Previous work shows that cortical VIP cells exhibit diverse

76 molecular, morphological, and electrophysiological properties. However, it is unknown whether

77 VIP cells have heterogeneous functions in the ACC and whether disinhibitory circuits are involved

78 in ACC processing of diverse stimuli.

79 We used miniscopes to perform in vivo single-cell resolution calcium imaging of VIP

80 interneurons in the ACC (VIP ${ }^{\mathrm{ACC}}$ ) to investigate their functional heterogeneity while mice

81 performed tasks to assay anxiety-like behaviors, general sociability and social novelty. We

82 identified distinct VIP ${ }^{\mathrm{ACC}}$ subgroups that reliably encoded behavioral states by preferentially

83 activating to anxiety-related, social, or non-social stimuli. In addition, we found that averaging

84 selective cell activity made this coding more reliable. These results suggest that the improvement

85 in coding was mediated through co-activation of these inhibitory subpopulations. When the same 
neurons were monitored across anxiety-related and social tasks, we determined that the majority

87 of VIP ${ }^{\mathrm{ACC}}$ that were engaged during these tasks were highly selective, activating to only one

88 specific stimulus. Lastly, using rabies trans-synaptic mapping, we showed that VIPACC receive

89 inputs from brain regions implicated in emotional regulation and social behavior. Our data show

90 that VIP ${ }^{\mathrm{ACC}}$ are functionally heterogeneous and that non-overlapping subgroups of VIPACC activate

91 preferentially, providing a cellular substrate for encoding different types of stimuli.

92

93 MATERIALS AND METHODS

95 Animals

96

97 Animals were grouped housed in a 12-hr light/dark schedule vivarium with food and water ad

98 libitum. Experimental mice were male postnatal day 60-120 VIP-Cre mice (Vip-IRES-cre,

$99 \#$ \#10908, The Jackson Laboratory, Bar Harbor, Maine (28)). Stimulus mice for social interaction

100 were littermates (male VIP-Cre) or novel (age matched male CD-1 IGS, strain code: 022, Charles

101 River Laboratories, Wilmington, Massachusetts). All procedures were approved by the

102 Institutional Animal Care and Use Committee (IACUC) at Boston University and practices were

103 consistent with the Guide for the Care and Use of Laboratory Animals and the Animal Welfare

104 Act.

105

106 Surgeries 
108 Surgeries were performed using aseptic surgical techniques with autoclaved instruments. Animals

109 were weighed and anesthesia was induced in a chamber with an isoflurane-oxygen mixture (4\%

$110[\mathrm{v} / \mathrm{v}])$. Anesthesia was maintained throughout the procedure via mask inhalation of an isoflurane-

111 oxygen mixture (Henry Schein, Melville, New York, 1-1.5\% [v/v]). Animals were kept on a

112 heating pad (T Pump, Gaymar Industries Inc., Orchard Park, New York) for the duration of the

113 surgeries and for 30-min recovery periods before being returned to their home cages. Animals

114 were injected with buprenorphine (3.25 mg/kg; SC, Patterson Veterinary, Greeley, Colorado),

115 meloxicam (5 mg/kg; SC, Covetrus, Dublin, Ohio), and dexamethasone (Henry Schein, $2.5 \mathrm{mg} / \mathrm{kg}$;

$116 \mathrm{SC}$ ) and the fur on the top of the head was removed. Animals were head-fixed using a stereotax

117 (Kopf Instruments, Tujunga, California). The surgical area was sterilized with 10\% povidone-

118 iodine and 70\% isopropyl alcohol (CVS, Woonsocket, Rhode Island) and local anesthetic was

119 applied (lidocaine 1\% and epinephrine 1:100,000; SC, Henry Schein). After surgeries, post-

120 operative analgesics were administered for 2 days, twice per day (buprenorphine $0.01 \mathrm{mg} / \mathrm{kg} ; \mathrm{SC}$

121 and meloxicam $5 \mathrm{mg} / \mathrm{kg}$; SC).

122 Viral injections: After the preparations above, an incision was made in the skin along the

123 midline of the skull. A craniotomy was made over the injection site using a pneumatic dental drill

124 (eBay, Inc., San Jose, California). Using a stereotax and the Nanoject II (Drummond Scientific,

125 Broomall, Pennsylvania), a pulled-glass pipette (BF150-117-10; tip size 3-15 $\mu$ m, Sutter

126 Instrument Co., Novato, California) was lowered into the ACC (AP: $+0.90 \mathrm{~mm}$, ML: $-0.30 \mathrm{~mm}$,

127 DV: $-1.00 \mathrm{~mm}$ ) and virus was injected. After pipette removal, the skin was sutured with non128 absorbable sutures (AD Surgical, Sunnyvale, California).

To monitor VIPACC activity, we injected $460 \mathrm{nl}$ of an adeno-associated virus (AAV) that 
131 GCaMP6f.SV40, titer: $5.23 \times 10^{13} \mathrm{GC} / \mathrm{ml}$, packaged by the University of Pennsylvania Vector 132 Core, Philadelphia, Pennsylvania). For trans-synaptic tracing of monosynaptic inputs to VIPACC, we first injected $128 \mathrm{nl}$ of a

134 helper AAV that expresses target proteins under the human synapsin-1 promoter: AAV2/1-synP135 Flex-splitTVA-EGFP-B19G (AAV-TVA-Glyco) (29) (titer: $0.98 \times 10^{12} \mathrm{GC} / \mathrm{ml}$, University of 136 North Carolina Viral Core, Chapel Hill, North Carolina). This AAV contained genes to express 137 EGFP, the avian sarcoma/leukosis virus subtype A receptor (TVA, which confers infection 138 capability to rabies virus pseudotyped with the avian sarcoma leucosis virus glycoprotein (EnvA)), 139 and the rabies virus glycoprotein $(\mathrm{G})(29,30)$ (which is necessary for trans-synaptic transport of 140 glycoprotein gene-deleted $(\Delta \mathrm{G})$ rabies virus $(\mathrm{RV}))(23)$. These three genes were in frame and 141 separated by porcine teschovirus self-cleaving 2A elements (29). After allowing the virus to 142 express for one month (31), the skin was re-incised, a new craniotomy was drilled, and $128 \mathrm{nl}$ of $143 \Delta \mathrm{G}$ EnvA pseudotyped RV with mCherry was injected (RVdG, titer: $1.5 \times 10^{9} \mathrm{GC} / \mathrm{ml}$, Boston's 144 Children Hospital Viral Core, Boston, Massachusetts). There is no cognate receptor for EnvA in 145 the mouse, so RVdG only infects TVA-expressing cells. Together, Glyco, TVA, and RVdG allow 146 for retrograde monosynaptic tracing only from Cre-expressing cells. In Cre-negative mice $(\mathrm{N}=6)$, 147 we performed these injections and saw no labeled cells, suggesting a lack of leaky expression (data 148 not shown). GRIN lens implants: To image neuronal activity with a miniaturized microscope 150 (miniscope), a gradient-index (GRIN) lens was implanted in the ACC at least 2 weeks after viral 151 injection (to allow for maximal GCaMP6f expression). After the preparations above, the scalp was 152 re-incised and a $1 \mathrm{~mm}$ diameter craniotomy was drilled, centered around the viral injection. Three 153 screws (Fine Science Tools Inc., North Vancouver, Canada) were inserted into the skull and a layer 
154 of super glue (cyanoacrylate, Krazy glue, High Point, North Carolina) was applied to ensure the

155 lens and dental cement adhered strongly. Dura over the ACC and a small region of the secondary

156 motor cortex were aspirated using a blunted $18 \mathrm{G}$ needle (BD, Franklin Lakes, NJ) coupled to a

157 vacuum line. A GRIN lens (Table S1) attached to a stereotax via custom 3D-printed implant

158 assembly (Fig S1) was lowered into the ACC at a $20^{\circ}$ angle (AP: $+0.90 \mathrm{~mm}, \mathrm{ML}:-0.12 \mathrm{~mm}, \mathrm{DV}$ :

$159-0.13 \mathrm{~mm})$, to improve access to the ACC and minimize the risk of puncturing the midline

160 vasculature. Lenses were adhered to the skull with optical glue (Norland Products Inc., Cranbury,

161 NJ) and dental cement (Ortho-Jet ${ }^{\mathrm{TM}}$ Liquid, Black, Lang Dental, Wheeling, Illinois). An antibiotic

162 was administered via the water supply (Biomox, $0.75 \mathrm{mgl} / \mathrm{ml}$, Henry Schein) for 10 days after

163 surgery.

\section{D-printed miniscopes and baseplating}

1673 weeks after lens implant surgeries (to allow for recovery and optimal GCaMP6f expression),

168 animals were anaesthetized, as described above, and GCaMP6f expression was assessed by

169 imaging the fluorescent signal using miniscopes. When GCaMP6f-positive neurons were visible,

170 baseplates were attached to the skulls with dental cement using a custom 3D printed baseplating

171 assembly (Fig S1E-F). The miniscopes used were modified from two existing designs (Ghosh et

172 al. 2011 (32), Liberti et al. 2017 (33)) to allow it to detach from the baseplate (Fig S1), which made

173 it possible to co-housed the animals without risking miniscope damage. Miniscopes were attached

174 to baseplates at a 15-20 degree angle relative to the midline to align with the GRIN lenses (Fig

175 S1B). Miniscope models are available at https://github.com/CruzMartinLab. Commercially 
available parts are listed in Table S1. Custom parts were 3D printed (Form 3 Printer, Black Resin

\section{Perfusions and Histology}

Animals were perfused to visualize viral injections and lens placements. Mice were injected with

Michigan) and transcardially perfused with phosphate buffered saline (PBS) followed by 4\% mounting medium with DAPI (Thermo Fisher Scientific, Waltham, Massachusetts) to visualize nuclei.

\section{$191 \quad$ Imaging}

193 Sections were imaged using an upright wide-field microscope (Nikon Eclipse Ni, Nikon

194 Instruments Inc. Melville, New York) controlled by NisElements (Nikon Instruments Inc., 4.20).

195 Images were acquired using a Plan Fluor 4X (NA 0.13) or 10X (NA 0.3) objective with standard

196 Nikon HQ filter cubes for DAPI, EGFP/GCaMP, and mCherry. Images were analyzed as TIFFs in

197 Image (National Institute of Health, Bethesda, Maryland) and compared to the Allen Mouse Brain 198 Atlas (34) (brain-map.org/api/index.html) to identify brain regions. 


\section{Behavioral assays and in vivo $\mathrm{Ca}^{2+}$ imaging}

Before any behavioral testing, mice were handled for 10 min for 3 days to acclimate them to the experimenter. For anxiety-related assays, mice were not exposed to the arenas prior to testing, but

204 for the social task, implanted mice were acclimated to the arena with empty cups for 10 min per for 10 min on each of these days. Arenas were custom made from acrylic and HDPE (McMaster-

214 LED power was adjusted to optimize imaging for each animal with a maximum output of $1 \mathrm{~mW}$.

215 To avoid bleaching GCaMP6f, no trials were longer than $10 \mathrm{~min}$.

$216 \quad$ Elevated zero maze (EZM): To assay anxiety-like behavior, we used the EZM, an elevated

217 circular arena with two open arms and two closed arms (track diameter $=50 \mathrm{~cm}$, track width $=5$

$218 \mathrm{~cm}$, wall height for closed arms $=40 \mathrm{~cm}$, height of track $=61 \mathrm{~cm})$. Open arms are considered 219 anxiogenic and closed are anxiolytic. Mice were initially placed in the closed arm and recorded 220 for $10 \min (36)$. 
Social task: The arena contained two mesh wire cups - one on each end of the arena $(50 \mathrm{x}$

223 and the other was empty. On Days 2 and 3 (Social Novelty), one cup housed a littermate and the

224 other housed an entirely novel male CD-1. Each day the experimental mouse was placed in the 225 center of the arena and given $10 \mathrm{~min}$ to explore the arena and cups. The side of the arena with each

226 stimulus was randomized.

Open field (OF): To assay anxiety-like behavior and locomotion, the OF was used. The center of the arena is considered anxiogenic and the periphery is anxiolytic. Mice were placed in the center of a custom-made acrylic arena $(50 \times 50 \times 30 \mathrm{~cm}$ length-width-height $)$ and allowed to 230 explore for $10 \mathrm{~min}$.

\section{Behavioral analysis}

234 Behavior was analyzed using DeepLabCut (37), an open-source program that uses machine

235 learning to track body parts in behavioral videos, as described by Comer et al. (38). To assess

236 accuracy, videos labeled by the software were inspected by a trained observer and custom

237 MATLAB (MathWorks, Natick, Massachusetts) scripts were used to verify that DeepLabCut

238 located each body part at least $95 \%$ of the total time the animal was tracked. For EZM and OF, we

239 tracked the centroid of the mouse's body to determine velocity, distance traveled, and when the

240 mouse was in each zone of the arena. For the $\mathrm{OF}$, we divided the arena into 25 squares $(10 \mathrm{~cm} \mathrm{x}$

$24110 \mathrm{~cm}$ each) and defined the periphery as the outermost 16 and the remaining 9 as the center. For

242 the social tasks, we tracked the head to determine close proximity to either cup. Binary behavior 
243 matrices (vectorized behavior) indicating the location of the animal were created from

244 DeepLabCut using custom MATLAB scripts.

\section{$246 \mathbf{C a}^{2+}$ imaging analysis}

$248 \mathrm{Ca}^{2+}$ imaging data were processed using CaImAn (39) written in Python

249 (https://www.python.org/). $\mathrm{Ca}^{2+}$ recordings underwent piecewise rigid motion correction using 250 patches of $48 \times 48$ pixels with $24 \times 24$ pixel overlap. Following image stabilization, cell detection 251 and extraction of $\mathrm{df} / \mathrm{f}$ traces were performed with a merging threshold of activity correlation greater 252 than 0.7 between nearby cells, and a 2.5 minimum threshold for the signal to noise ratio. $\mathrm{df} / \mathrm{f}$ traces 253 and spatial information were exported and saved in .mat format using SciPy (40). All subsequent 254 analysis was performed using custom MATLAB and Python functions. Raw $\mathrm{Ca}^{2+}$ traces were z255 scored using the mean baseline $\mathrm{df} / \mathrm{f}$ and sigma from the entire time series for each trial. Z-scored 256 traces report deltaf/f (df/f) values in units of standard deviation (SD). Due to the different 257 acquisition frequencies of behavioral and $\mathrm{Ca}^{2+}$ imaging videos, data were aligned using timestamps 258 from acquisition and custom MATLAB scripts. Gaps in neural data due to dropped frames (less 259 than $1 \%$ of the total frames) were filled with averaged $\mathrm{z}$-scored $\mathrm{df} / \mathrm{f}$ values from surrounding 260 frames. Starts and ends of behavioral epochs were matched to $\mathrm{Ca}^{2+}$ data timestamps to isolate 261 neural activity during select behaviors.

262 Calcium activity: For all figures, $\mathrm{Ca}^{2+}$ activity refers to the area under the curve. To 263 quantify this value, we used z-scored $\mathrm{df} / \mathrm{f}$ traces and took their integral using the MATLAB trapz 264 function. For all figures, excluding Fig S2I-J, area under the curve was calculated for $5 \mathrm{~s}$ intervals, 265 which was chosen based on average transient length. When calculating the average area under the 
curve across velocities (Fig S2I-J), the intervals were $1 \mathrm{~s}$. These integral values were calculated

267 for each cell to get the $\mathrm{Ca}^{2+}$ activity values. When average $\mathrm{Ca}^{2+}$ activity is reported, that refers to

268 the average area under the curve for all cells for each mouse.

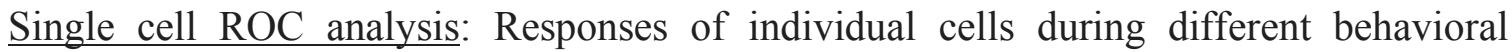
conditions were assessed within each behavioral trial using receiver operating characteristic (ROC)

271 analysis, as previously described (41). The ROC curve demonstrates how well a single neuron's

272 activity matches an animal's behavioral state, which can be quantified by calculating the area under

273 the ROC curve (auROC) $(41,42)$. For each neuron in each behavioral condition, an ROC curve 274 was generated using the true positive rate (TPR) and false positive rate (FPR) values for that cell 275 and behavioral state. TPR and FPR were calculated across multiple binary thresholds applied to z276 scored $\mathrm{df} / \mathrm{f}$ traces of each cell, ranging from the minimum to maximum values of the $\mathrm{Ca}^{2+}$ signal.

277 For each threshold, binarized df/f traces were compared to the binary behavioral vectors, which 278 used binary values to indicate an animal's presence or absence in a specific zone of the arena. TPR 279 and FPR were then plotted against each other to create each ROC curve and auROC was calculated. To classify cells as stimulus-selective or neutral, we determined whether the cell's auROC 281 value for a given stimulus was high enough to suggest it preferentially activated to a stimulus. To 282 do this while accounting for any random alignment in our data, we calculated 1000 null values for 283 each cell by applying circular permutations of randomized lengths to the $\mathrm{Ca}^{2+}$ data and calculating 284 auROC for each of these randomized versions of the data. Cells were considered selective for a 285 certain stimulus if the auROC of that cell was at least 2 SD greater than the mean of the null 286 distribution (auROC $>97.5^{\text {th }}$ percentile). Otherwise, cells were considered neutral. For Fig 3, to 287 calculate the auROC of "super cells", we averaged the z-scored df/f traces of all selective cells and 288 re-calculated auROC for that averaged data. 
auROC analysis across tasks: For Fig 4A-D, to determine if the same cells were responsive

291 (127 registered VIPACC from all 6 animals). For each cell and behavioral condition, auROC was

292 calculated and cells were considered selective for multiple conditions (as described above) if they

293 were selective for different stimuli across these tasks.

using the first half of the EZM or Sociability data, rather than the entire dataset, to identify selective context or stimulus in that task. the closest timestamp.

\section{Analysis of rabies tracing data}

308 For retrograde mapping experiments, brains were scanned to identify signal from starter and 
312 animal had a different number of starter cells, so to normalize our data, we divided the number of

313 retrogradely-labeled neurons in each region by the number of starter cells for that mouse (Inputs

314 per starter cell). The number and location of labeled neurons in a given region was independently

315 confirmed by 3 trained scientists. After quantifying all cells, input brain regions were divided into

316 quartiles by number of input neurons. Only brain regions in the top two quartiles were graphed

317 and included in Fig 4. We did not include RVdG inputs at the site of the ACC injection since

318 leakage in TVA expression could lead to Cre-independent local labeling (43).

\section{Statistical analysis}

Statistical analyses were performed using Graph Pad Prism 8.0 (GraphPad Software Inc., San

Ottawa, Canada) and ImageJ were used. The threshold for significance was set to $\alpha=0.05$ and ${ }^{*} p<0.05, * * p<0.01, * * * p<0.001, * * * * p<0.0001$. Data are presented as mean $\pm \mathrm{SEM}$, unless otherwise noted. t-tests and ANOVAs followed by appropriate post tests were used and are specified in the figure legends. For Fig S3A-B, Fig S4K-L, and Fig S6A-D, frequency distributions were fitted with Gaussians and percentages of selective cells are represented in pie charts. For all

329 behavioral experiments, $\mathrm{N}=6$ implanted mice for $\mathrm{Ca}^{2+}$ imaging and $\mathrm{N}=5$ control mice that 330 underwent no surgeries. For EZM, $n=345$ cells, for Sociability, $n=310$ cells, for Social Novelty 331 Day 2, $n=350$ cells, Day $3, n=232$ cells, and OF, $n=273$ cells. For tracing experiments, $N=3$ mice with $n=691$ starter cells and 10107 retrogradely-labeled input cells. 


\section{Distinct VIP ${ }^{\mathrm{ACC}}$ interneurons preferentially encode anxiogenic or anxiolytic contexts}

To determine whether VIP ${ }^{\mathrm{ACC}}$ are a heterogeneous population, we imaged their activity as mice

341 injections and lenses were successfully targeted to the ACC (Fig 1B), allowing monitoring of

342 VIPACC with single-cell resolution during behavioral assays (Fig S2A, Fig 1C). 3D-printed each VIP ACC and performed ROC analysis ((41)Fig 1D). auROC values quantify how well each

352 cell's activity matches a behavioral state; values near 0.5 are expected for cells that do not encode

353 behavioral states and higher auROCs reflect better encoding of this information. For each neuron,

354 we calculated an auROC for the closed arms and an auROC for the open arms. We identified individual VIPACC that demonstrated selectively increased activity in one arm type and classified 
states, as compared to neutral cells (Fig 1D). Closed-selective cells showed increased activity

while animals were in the closed, as compared to the open arms (Fig 1E, top and G), whereas openselective cells showed the opposite effect (Fig 1E, bottom and $\mathrm{H}$ ).

To determine whether selective VIPACC activity rapidly changes in anxiolytic or anxiogenic contexts, we isolated trials when animals transitioned from one arm to the other (Fig 1J) and plotted

363 VIPACC activity. Heatmaps and $\mathrm{Ca}^{2+}$ activity showed robust differences during behavioral

364 transitions (Fig 1K-R). Closed-selective cells preferentially activated soon after animals entered 365 the closed arms $(27 \%$ increase, Fig $1 \mathrm{~K}$ and O) or prior to entering the open arms ( $45 \%$ increase, 366 Fig 1L and P). Open-selective cells were preferentially active soon after animals transitioned into 367 the open arms (22\% increase, Fig $1 \mathrm{M}$ and Q) or before leaving the closed arms (44\% increase, Fig $3681 \mathrm{~N}$ and $\mathrm{R})$.

We verified that this analysis method was reliable by using half of our data to identify 370 selective cells and using those classifications to perform $\mathrm{Ca}^{2+}$ activity analysis on the remaining 371 half (Fig S5A). In this second half of the data, activity of selective cells increased when mice were 372 in the preferred context, relative to the non-preferred (23\%, Fig S5B). Additionally, the majority 373 of auROC values were near 0.5 for both zones (Fig S3A-B), suggesting that most VIP ${ }^{\text {ACC }}$ were 374 neutral and did not preferentially activate to a specific context (Fig 1I, Fig S3C).

375 We next assessed whether these effects were context-specific, or they could also be 376 identified in a second task of anxiety-like behavior. Similar to our findings in the EZM, we found 377 that mice in the open field (OF) avoided the anxiogenic center zone (Fig S2C and D) and we 378 identified zone-specific cells that encoded the animal's behavioral state (Fig S4). In contrast to the 379 OF, in the EZM there was no relationship between the animal's velocity and VIPACC activity (Fig 380 S2I and J), which suggests that the activity differences in EZM reflect the animal's anxiogenic 
381 state, rather than signals related to locomotion. These data suggest that our selectivity

382 classifications accurately reflect the relationship between VIP ${ }^{\mathrm{ACC}}$ activity and anxiety-related

383 behaviors.

384 Despite differences in behavior and the activity of individual neurons, there was no 385 difference in the average activity of all VIP ${ }^{\mathrm{ACC}}$ as mice explored different zones of the EZM or OF 386 (Fig S2B and G-H), which suggests that VIP ${ }^{A C C}$ do not uniformly activate in anxiogenic or 387 anxiolytic contexts. Additionally, the behavior of implanted, miniscope-mounted animals was 388 compared to control mice and no behavioral differences were found, suggesting that neither 389 surgeries nor implants induced changes in locomotion or anxiety-like behaviors (Fig S2K-N). 390 Overall, these results support our hypothesis that VIPACC are a heterogeneous population, where 391 subpopulations preferentially activate in either anxiolytic or anxiogenic contexts.

393 Distinct subpopulations of VIP ${ }^{A C C}$ encode social and non-social stimuli

395 We next investigated whether individual VIP ${ }^{A C C}$ were selective for social or non-social stimuli by 396 recording their activity as mice interacted with these stimuli over three days (Fig 2A and D). In 397 Sociability, mice explored a chamber with an empty mesh cup (which they had been acclimated 398 to) and a littermate in a mesh cup; in Social Novelty, the cups housed either a littermate or a novel 399 mouse. Implanted mice spent more time with their littermate than an empty cup (Fig 2B, 2.4 fold400 change), and more time with the novel mouse than the littermate (Fig 2C, 1.8 fold change), 401 suggesting an interest in socialization and a preference for novel social stimuli. Using ROC analysis, we identified cup-, littermate-, and novel-mouse-selective cells and 
404 Sociability, cells were classified as cup-selective (15\%), littermate-selective (17\%), selective for 405 both stimuli (2\%), or neutral (Fig 2D-G). For Social Novelty, cells were classified as littermate406 selective (11\% Day 2, 20\% Day 3), novel mouse-selective (24\%, 12\%), or neutral (64\%, 68\%, Fig 407 2D-G). Very few cells were selective for both stimuli (Fig 2G, 0-1\% of cells, Littermate- and cup408 selective or littermate- and novel mouse-selective cells). Littermate-selective cells showed 409 increased activity while animals interacted with the littermate, as compared to the cup (36\% 410 increase, Fig 2F, top and $\mathrm{H}$ ) or novel mouse (33\% increase, Fig 2J). Cup-selective VIP ${ }^{\mathrm{ACC}}$ were 411 more active when animals interacted with the cup than with the littermate (40\% increase, Fig $2 \mathrm{~F}$, 412 middle and I). Novel mouse-selective neurons demonstrated increased activity while mice 413 interacted with novel mice than with littermates (36\% increase, Fig $2 \mathrm{~K}$ ). Similar to the OF and 414 EZM (Fig 1I, Fig S3 and Fig S4J-M), most cells were classified as neutral and their activity did 415 not change as mice interacted with various stimuli (Fig 2F, bottom and Fig S6A-F). We also 416 verified that this analysis method was reliable as described above (Fig S5A) and the $\mathrm{Ca}^{2+}$ activity 417 of selective cells increased when mice interacted with preferred stimuli (preferred vs. non418 preferred, 17\%) (Fig S5C). Similar to the anxiety-related assays, we found no differences in the

419 average activity of all VIP ${ }^{A C C}$ as mice interacted with the different social or non-social stimuli (Fig 420 S6G-H). This suggests that there is no global change in the VIP ${ }^{A C C}$ activity during interactions 421 with other mice or objects. These data also suggest that, in addition to encoding for anxiogenic 422 and anxiolytic contexts, distinct subgroups of VIP ${ }^{A C C}$ encode for interactions with objects, other 423 mice, and social novelty. 
427 Our analysis thus far demonstrated that individual VIPACC can reliably encode diverse stimuli. To

428 determine whether subsets of selective VIP ${ }^{\mathrm{ACC}}$ encode behavioral states as a population to increase

429 the reliability of their information, we averaged the $\mathrm{Ca}^{2+}$ traces from all cells that were selective

430 for a specific stimulus to create "super cells" (Fig 3A-D). This process determines whether

431 selective cells are co-activating or whether they primarily activate asynchronously.

432 Unsynchronized $\mathrm{Ca}^{2+}$ transients in averaged traces would cancel each other out, resulting in

433 auROC values similar to individual selective cells. If cells co-activate, however, the auROC values

434 of averaged traces would be significantly higher than individual auROC values. We compared

435 super cell auROC values to those from all VIP ACC (Fig 3A, obtained by averaging the auROC

436 values for all cells) and from the selective cells (obtained by averaging the individual auROC

437 values of selective cells). In the EZM, the auROC values for closed super cells increased by $34 \%$

438 and $12 \%$ relative to all or closed-selective VIP ${ }^{A C C}$, respectively (Fig 3B, E). Similarly, for open

439 super cells, we found increases of $42 \%$ and $19 \%$ in auROC values relative to all or open-selective

440 VIP ${ }^{\mathrm{ACC}}$, respectively (Fig 3B, F). We found this pattern in other behavioral tasks, as well. In

441 Sociability, littermate super cells exhibited a $24 \%$ and $11 \%$ increase in auROC values relative to

442 all or littermate-selective VIPACC, respectively (Fig 3C, G). In Social Novelty, auROC values

443 increased by $27 \%$ and $10 \%$ relative to all or littermate-selective VIP ${ }^{\mathrm{ACC}}$, respectively (Fig $\left.3 \mathrm{D}, \mathrm{I}\right)$.

444 Cup super cell auROCs increased by $29 \%$ and $13 \%$ relative to all or cup-selective VIPACC,

445 respectively (Fig 3C, H), and novel mouse super cells exhibited $24 \%$ and $11 \%$ increases in auROC

446 values relative to all or novel-mouse-selective VIP ${ }^{\mathrm{ACC}}$, respectively (Fig 3D, J). Overall, these data

447 support previous findings and demonstrate that averaging the activity of stimulus-selective VIPACC

448 increases the accuracy of their coding, suggesting that these cells encode information as

449 disinhibitory subclusters. 


\section{VIP ${ }^{A C C}$ subpopulations are non-overlapping and are recruited during distinct behaviors}

453 To determine whether diverse neuronal representations are embedded in particular VIP ACC $^{\text {AC }}$

454 subpopulations, we monitored the same cells across tasks (Fig 4A and B). By registering our neural

455 data, we identified the same 127 neurons across tasks (out of 655 total neurons) and tracked their

456 activity in both EZM and Sociability (Fig 4A and B). We identified distinct subpopulations of

457 VIP ${ }^{\mathrm{ACC}}$ that showed stimulus selectivity in only one task and others that were selectively active in

458 specific zones of each task (Fig 4C). $27 \%$ of registered VIP ${ }^{\mathrm{ACC}}$ were selective in the EZM and

459 neutral during Sociability (Fig 4C). Similarly, about 22\% were selective in Sociability, but neutral

460 in the EZM (Fig 4C). Out of all registered VIPACC, 17\% were only open-selective, 9\% only closed-

461 selective, $16 \%$ only littermate-selective, and $6 \%$ only cup-selective. Taken together, these data

462 show that about half of all registered VIPACC were only selective for one of these 4 stimuli, whereas

463 only one sixth were selective for 2 or 3 stimuli (e.g., both when the mouse was in the open arm of

464 the EZM and when it interacted with the littermate in Sociability) (Fig 4C). This demonstrates that,

465 on average, more cells were selective for one stimulus than for two or three (Fig 4C-D). Unlike in

466 the previous data without registration, where the majority of cells were neutral, only $36 \%$ of cells

467 were neutral in both tasks (Fig 4C). These data suggest that, within the ACC, there are non-

468 overlapping VIP interneuron subcircuits dedicated to processing particular stimuli. 
472 To determine whether VIP ${ }^{\mathrm{ACC}}$ receive projections from other brain areas involved in anxiety and 473 social behavior, we used rabies virus-mediated trans-synaptic mapping. This technique allowed us 474 to retrogradely label only neurons that synapse onto VIP ${ }^{A C C}$ (Fig 4E-J). First, we injected AAV475 TVA-Glyco-GFP, which expresses target proteins in a Cre-dependent manner, in VIP-Cre mice to 476 target VIP ${ }^{\mathrm{ACC}}$. Four weeks later, we injected RVdG, such that starter neurons expressed both EGFP 477 (pseudo colored magenta, also expressing TVA and $\Delta \mathrm{G}$ ) and mCherry (pseudo colored yellow, 478 infected by RVdG) and input cells only expressed mCherry (Fig 4E and F). We identified these 479 labeled cells, quantified their numbers in each brain region (Fig 4E and F), and normalized input 480 cells to starters. We found retrogradely-labeled neurons were most prominent in other regions of 481 the PFC (about 18\% of all labeled regions), primary and associative areas (prim/asso), thalamic 482 nuclei, and the medial septal complex (MSC), suggesting that these regions were highly connected 483 to $\mathrm{VIP}^{\mathrm{ACC}}$ (Fig 4F). We further partitioned the regions with the greatest number of retrogradely484 labeled neurons into subregions and determined that VIPACC received connections from the 485 contralateral (cl) ACC (77\% of retrogradely-labeled PFC neurons, Fig 4H), prelimbic cortex (PrL, $48612 \%$ of retrogradely-labeled PFC neurons, Fig 4H), retrosplenial cortex (RS, including RS granular 487 (RSG) and dysgranular (RSD), 86\% of retrogradely-labeled prim/asso neurons, Fig 4I), 488 anteromedial thalamic nucleus (AM) (46\% of retrogradely-labeled thalamic neurons, Fig $4 \mathrm{~J})$, and 489 lateral posterior thalamic nucleus medio rostral part (LPMR, 8\% of retrogradely-labeled thalamic 490 neurons, Fig 4J). Our data showed that VIPACC receive long-range projections, not only from 491 regions implicated in emotional regulation and social cognitive behavior, but also areas important 492 for neuromodulation, memory formation, and motor actions. 
Multimodal disinhibitory circuits in the ACC

498 Like many prefrontal cortical regions, the ACC is implicated in a wide range of behavioral

499 functions. Our work supports past findings by connecting ACC activity with anxiety-related and

500 social behavior and identifies a neural substrate for processing of multimodal stimuli. However,

501 an important facet of our finding is that a diverse range of neuronal representations are encoded in

502 subgroups of VIP ${ }^{\mathrm{ACC}}$, which points to the varied roles of the ACC.

Human imaging studies have shown that ACC activity increases as individuals perform social and emotional tasks, as compared to non-social tasks (44). Interestingly, this previous work demonstrates heterogeneous activation of different subregions within the ACC, where increased activity of the perigenual ACC and rostral ACC is implicated in social tasks, whereas dorsal ACC

507 is selectively activated in non-social cognitive tasks (44). Our histological analysis revealed that

508 lenses were located at the border of the dorsal and ventral subregions of the ACC (A24a and A24b

509 subregions). Future research could monitor VIP cells in different ACC subregions to determine

510 whether the selectivity distributions differ. Additionally, we found subgroups of VIPACC that

511 encoded interactions with objects, which supports past findings that characterized neural correlates

512 in the ACC for object and location recognition and memory consolidation of object/place

513 associations $(14,15)$. Our work shows that approximately $70 \%$ of VIP ${ }^{A C C}$ were neutral in the

514 anxiogenic and social behavioral tasks. Perhaps this high prevalence of neutral cells reflects the 515 multimodal nature of the ACC, where these cells encode other kinds of stimuli. For example, 516 previous work highlighted the role of the ACC in pain processing (45). Alternatively, cells that 517 were classified as neutral may be equally activated by multiple stimuli. For example, neutral EZM 
518 cells may preferentially activate to both anxiogenic and anxiolytic stimuli and be involved in 519 encoding both.

When we averaged the activity of stimulus-selective VIP ACC, the reliability of their coding

521 increased across diverse stimuli. Electrical coupling has been observed within inhibitory networks,

522 including VIP interneurons $(22,46)$. VIP cells can disinhibit members of their own population,

523 leading to increased co-activation of the population, which may allow these subnetworks to encode

524 stimuli as a population and amplify the population output (22).

\section{Whole brain mapping of long-range direct inputs to VIPACC}

528 Using rabies trans-synaptic mapping, we provided a brain-wide map of inputs to VIP ${ }^{\mathrm{ACC}}$. 529 Additionally, we demonstrated that $\mathrm{VIP}{ }^{\mathrm{ACC}}$ receive extensive connections from the AM and RS,

530 which are both known to project to the ACC (47-50). AM lesions are associated with memory 531 impairments (51-53), so AM-ACC projections may be important for social memory. In support of 532 this, mice lacking Shank3 in the ACC show abnormal behavior in a social novelty task (8).

533 Additionally, memory formation leads to induction of immediate-early genes in the ACC (54). In

534 the auditory cortex, VIP cells facilitate learning about unexpected, salient events (55), suggesting 535 that inhibitory circuits in the ACC could mediate specific aspects of memory formation. Although 536 it remains to be tested, AM-VIPACC projections are positioned to mediate social learning and 537 memory. In rodents, AM-ACC projections regulate histaminergic itch-induced scratching 538 behavior (56), suggesting that, similar to sensory thalamocortical projections, this higher-order 539 network could be composed of thalamic pathways for parallel processing (31). The RS plays a role 540 in learning, memory, and navigation and is highly connected to the anterior thalamic nuclei and 
541 hippocampus $(51,57-59)$. Spatial information conveyed to VIPACC from the RS could guide motor

542 actions during social behavior $(58,60)$. We also showed that VIP ${ }^{\mathrm{ACC}}$ are highly connected to other

543 prefrontal cortex subregions and the contralateral ACC. Our results support human imaging studies

544 demonstrating that distinct ACC-PFC networks are involved in diverse aspects of emotional

545 processing (1) and monitor behavior to guide compensatory systems (61).

546 Our injections and lenses were located at the border between the A24a and A24b

547 subregions of the ACC $(62,63)$. Past research shows that A24 receives projections from the

548 amygdala, orbitofrontal cortex, thalamus, RS, and motor cortex and moderate projections from

549 hippocampus, hypothalamus, and autonomic brain nuclei (63-65). Although our data is mostly

550 consistent with these findings, a major difference is that we used trans-synaptic viral mapping in

551 a cell-type specific manner, while other studies used classic neuronal tracers to map connectivity

552 to all ACC neurons (63-65). Mapping experiments that include inputs to other interneuron

553 subtypes will provide a better framework to understand ACC function and the connectivity patterns

554 of inhibitory circuits of the ACC.

\section{Heterogeneous inhibitory subcircuits in the ACC}

558 VIP interneurons express a variety of neuromodulator receptors, which may allow long-range

559 projections to exert context-dependent disinhibition $(19,20,55,66-69)$. Our data showed that 560 subgroups of VIP ${ }^{\mathrm{ACC}}$ are engaged by particular stimuli. Through the actions of neuromodulators, 561 VIP interneurons in the ACC may recruit subpopulations of Pyr that are behaviorally relevant or 562 encode specific information. 

coeruleus (LC) neurons, the primary source of norepinephrine in the forebrain, are recruited by novel stimuli, so this neuromodulator may be important in social novelty (70-73). Additionally, mice exhibit elevated arousal when exposed to novel or social stimuli, which is abolished upon lesioning of the ACC or LC (74). This suggests that noradrenergic modulation of the ACC may be

568 a substrate for attention and novelty (74). In the cortex, norepinephrine differentially regulates the activity of interneurons $(75,76)$, but it is unknown whether recruitment of VIPACC by novel social

570 stimuli is dependent on particular neuromodulators. We show that a small number of VIP ${ }^{\mathrm{ACC}}$ are 571 selective to multiple stimuli or contexts. It is possible that through the actions of neuromodulators, 572 distinct groups of VIPACC could overlap in their activity, allowing for the co-activation of 573 segregated Pyr subgroups (77). This cellular mechanism could enable ACC networks to bind 574 multiple streams of information to guide behavioral actions or monitor optimal performance (60, $57561,78)$. In the barrel cortex, the majority of VIP interneurons are located in layer $2 / 3$, but those in 576 other cortical layers exhibit different dendritic and axonal morphology (79). Due to the large field 577 of view in our $\mathrm{Ca}^{2+}$ imaging experiments and variability in lens placement, it was not possible to 578 correlate the activity of VIP to different cortical layers.

Using in vivo imaging with cellular resolution in freely moving mice, we showed that $580 \mathrm{VIP}{ }^{\mathrm{ACC}}$ are functionally heterogeneous, where distinct subcircuits encode for diverse stimuli. ROC 581 analysis identified stimulus-selective VIPACC that encoded anxiety-like behaviors or interactions 582 with mice and objects, even though there was no difference in overall VIP ${ }^{\mathrm{ACC}}$ activity. Averaging 583 the activity of selective VIPACC enhanced their ability to encode for specific stimuli. We also 584 determined that most VIP ${ }^{\mathrm{ACC}}$ were either selective for only one stimulus or were neutral. Lastly, 585 we used trans-synaptic mapping to provide a map of VIP ${ }^{\mathrm{ACC}}$ connectivity and show that VIP ${ }^{\mathrm{ACC}}$ 
receive inputs from regions implicated in emotional regulation, social cognition, and memory

587 formation. To our knowledge, these data provide the first evidence of functional heterogeneity of

588 VIPACC in vivo and show that population coding of selective VIPACC may encode stimulus-specific

589 information, which provides a framework for how the ACC encodes information across diverse

590 behavioral states.

\section{ACKNOWLEDGEMENTS}

594 We thank all research assistants in the Cruz-Martín lab, as well as Margaret Minnig, Timothy

595 Otchy, Nathan Perkins, and Daniel P. Leman for optimization of miniscope imaging and lens

596 implant placement, Tim Gardner for providing access to 3D printers and sharing video acquisition

597 software, Peyman Golshani and Daniel Aharoni for donating the camera sensor and data 598 acquisition board for miniscope experiments. Ashley Comer, Nancy Padilla, Mark Howe, William

599 A. Liberti 3rd and members of the Cruz-Martín lab helped critically by reading the manuscript and 600 engaging in helpful discussions. Todd Blute and the Boston University Biology Imaging Core 601 provided use of the epifluorescence microscope. Lastly, we used the Boston University Shared 602 Computing Cluster to analyze our data. This work was supported by a NARSAD Young 603 Investigator Grant (AC-M, \#27202), the NSF NRT UtB: Neurophotonics National Research 604 Fellowship (LNK, \#DGE-1633516), and the Boston University Undergraduate Research 605 Opportunities Program (TPHN, WWY) (https://www.bbrfoundation.org/grants-prizes/grants, 606 https://www.nsf.gov/; http://www.bu.edu/urop/). The funders had no role in study design, data 607 collection and analysis, decision to publish, or preparation of the manuscript. 
CONTRIBUTIONS

610

611 Connor Johnson: Conceptualization: formulated ideas for data analysis (equal), Software: code

612 writing and analysis for behavioral and calcium imaging data, calcium activity analysis with

613 CaImAn (lead). Formal Analysis: analysis of calcium data (equal). Investigation: surgeries,

614 histology, episcope imaging, cell counting (equal). Validation: validation of calcium analysis

615 strategy (lead). Writing - Original Draft: part of methods (support). Writing - Review \& Editing:

616 provided edits (equal). Visualization: figure generation (support).

617 Lisa N. Kretsge: Conceptualization: formulated composition and goals of the paper (equal),

618 Software: behavioral analysis with DeepLabCut (equal). Formal Analysis: histological analysis,

619 analysis of trans-synaptic tracing data, statistical analyses (equal). Investigation: behavioral

620 experiments, histology, episcope imaging (equal), Writing - Original Draft: wrote initial draft

621 (excluding part of methods and discussion) (lead). Writing - Review \& Editing: incorporated edits

622 (lead). Visualization: figure generation (equal).

623 William W. Yen: Methodology: miniscope adaptation design and construction, developed

624 protocols for GRIN lens implant surgeries (lead). Investigation: surgeries and baseplating,

625 behavioral experiments with miniscopes (lead). Writing - Review \& Editing: provided edits

626 (support). Visualization: figure generation (support).

627 Balaji Sriram: Conceptualization: provided ideas for analysis (support). Investigation: set up

628 DeepLabCut with our data (support). Supervision: oversight of some analysis projects (equal).

629 Jessica C. Jimenez: Methodology: taught our lab to perform surgeries and use miniscopes

630 (equal). Writing - Review \& Editing: provided edits (equal). 
631 Tushare J. Jinadasa: Formal Analysis: quantification of trans-synaptic tracing data (equal).

632 Investigation: surgeries, histology, episcope imaging, cell counting (equal). Writing - Review \&

633 Editing: provided edits (support). Visualization: figure generation (support).

634 Alexandra O'Connor: Software: code writing and analysis of the output of DeepLabCut data 635 (equal).

636 Ruichen Sky Liu: Software: code writing for calcium imaging analysis (support). Writing -

637 Original Draft: part of methods (support). Visualization: activity heatmaps figure generation 638 (support).

639 Thanh P. H. Nguyen: Methodology: miniscope adaptation design and construction (equal).

640 Eun Seon Cho: Investigation: annotation for behavioral analysis in DeepLabCut (support).

641 Erelle Fuchs: Investigation: surgeries, histology, episcope imaging, cell counting (support).

642 Eli D. Spevack: Investigation: histology, episcope imaging, cell counting (support).

643 Berta Escude Velasco: Investigation: cell counting (support). Writing - Review \& Editing:

644 provided edits (support).

645 Frances S. Hausmann: Investigation: perfusions and histology (support).

646 Alberto Cruz-Martín: Conceptualization: formulated composition, goals, and scope of the paper 647 and approaches for analyses (lead), Formal Analysis: statistical analyses (equal). Writing -

648 Original Draft. Writing - Review \& Editing: editing and feedback throughout (lead).

649 Visualization: figure design and generation (lead). Supervision: mentorship and oversight of the 650 project (lead). Project Administration: management and coordination (lead). Funding acquisition 651 (lead). 
655 The authors declare no conflicts of interest in this work.

656

\section{REFERENCES}

658

659 1. Etkin A, Egner T, Peraza DM, Kandel ER, Hirsch J. Resolving emotional conflict: a role

660 for the rostral anterior cingulate cortex in modulating activity in the amygdala. Neuron.

$661 \quad 2006 ; 51(6): 871-82$.

662 2. Kennerley SW, Walton ME, Behrens TE, Buckley MJ, Rushworth MF. Optimal decision 663 making and the anterior cingulate cortex. Nat Neurosci. 2006;9(7):940-7.

664 3. Lieberman MD, Eisenberger NI. The dorsal anterior cingulate cortex is selective for pain:

665 Results from large-scale reverse inference. Proc Natl Acad Sci U S A. 2015;112(49):15250-5.

666 4. Marusak HA, Thomason ME, Peters C, Zundel C, Elrahal F, Rabinak CA. You say

667 'prefrontal cortex' and I say 'anterior cingulate': meta-analysis of spatial overlap in amygdala-to-

668 prefrontal connectivity and internalizing symptomology. Transl Psychiatry. 2016;6(11):e944.

$6695 . \quad$ Rolls ET. The cingulate cortex and limbic systems for action, emotion, and memory.

$670 \quad$ Handb Clin Neurol. 2019;166:23-37.

671 6. Brockett AT, Tennyson SS, deBettencourt CA, Gaye F, Roesch MR. Anterior cingulate

672 cortex is necessary for adaptation of action plans. Proc Natl Acad Sci U S A.

$673 \quad 2020 ; 117(11): 6196-204$.

$6747 . \quad$ Nitschke JB, Sarinopoulos I, Oathes DJ, Johnstone T, Whalen PJ, Davidson RJ, et al.

675 Anticipatory activation in the amygdala and anterior cingulate in generalized anxiety disorder 676 and prediction of treatment response. Am J Psychiatry. 2009;166(3):302-10. 
677 8. Guo B, Chen J, Chen Q, Ren K, Feng D, Mao H, et al. Anterior cingulate cortex

678 dysfunction underlies social deficits in Shank3 mutant mice. Nat Neurosci. 2019;22(8):1223-34.

679 9. Rudebeck PH, Buckley MJ, Walton ME, Rushworth MF. A role for the macaque anterior

680 cingulate gyrus in social valuation. Science. 2006;313(5791):1310-2.

681 10. Rudebeck PH, Putnam PT, Daniels TE, Yang T, Mitz AR, Rhodes SE, et al. A role for 682 primate subgenual cingulate cortex in sustaining autonomic arousal. Proc Natl Acad Sci U S A. $683 \quad 2014 ; 111(14): 5391-6$.

684 11. Weible AP, Piscopo DM, Rothbart MK, Posner MI, Niell CM. Rhythmic brain 685 stimulation reduces anxiety-related behavior in a mouse model based on meditation training.

686 Proc Natl Acad Sci U S A. 2017;114(10):2532-7.

687 12. Kim SS, Wang H, Li XY, Chen T, Mercaldo V, Descalzi G, et al. Neurabin in the 688 anterior cingulate cortex regulates anxiety-like behavior in adult mice. Mol Brain. 2011;4:6.

689 13. Totah NK, Kim YB, Homayoun H, Moghaddam B. Anterior cingulate neurons represent 690 errors and preparatory attention within the same behavioral sequence. J Neurosci.

$691 \quad 2009 ; 29(20): 6418-26$.

692 14. Weible AP, Rowland DC, Monaghan CK, Wolfgang NT, Kentros CG. Neural correlates 693 of long-term object memory in the mouse anterior cingulate cortex. J Neurosci.

$694 \quad 2012 ; 32(16): 5598-608$.

695 15. Weible AP, Rowland DC, Pang R, Kentros C. Neural correlates of novel object and novel 696 location recognition behavior in the mouse anterior cingulate cortex. J Neurophysiol. $697 \quad 2009 ; 102(4): 2055-68$. 
698 16. Xiao Z, Martinez E, Kulkarni PM, Zhang Q, Hou Q, Rosenberg D, et al. Cortical Pain

699 Processing in the Rat Anterior Cingulate Cortex and Primary Somatosensory Cortex. Front Cell

$700 \quad$ Neurosci. 2019;13:165.

701 17. Askew CE, Lopez AJ, Wood MA, Metherate R. Nicotine excites VIP interneurons to

702 disinhibit pyramidal neurons in auditory cortex. Synapse. 2019;73(9):e22116.

703 18. Lee S, Kruglikov I, Huang ZJ, Fishell G, Rudy B. A disinhibitory circuit mediates motor

704 integration in the somatosensory cortex. Nat Neurosci. 2013;16(11):1662-70.

705 19. Pi HJ, Hangya B, Kvitsiani D, Sanders JI, Huang ZJ, Kepecs A. Cortical interneurons

706 that specialize in disinhibitory control. Nature. 2013;503(7477):521-4.

707 20. Sarah Melzer EN, Grace Or Mizuno, Minsuk Hyun, Adrienne C. Philson, Eleonora

708 Quiroli, Beatrice Righetti, Malika R. Gregory, Kee Wui Huang, James Levasseur, Lin Tian,

709 Bernardo L. Sabatini. Bombesin-like peptide recruits disinhibitory cortical circuits and enhances

710 fear memories. bioRxiv. 2020.

711 21. Karnani MM, Jackson J, Ayzenshtat I, Hamzehei Sichani A, Manoocheri K, Kim S, et al.

712 Opening Holes in the Blanket of Inhibition: Localized Lateral Disinhibition by VIP Interneurons.

713 J Neurosci. 2016;36(12):3471-80.

714 22. Karnani MM, Jackson J, Ayzenshtat I, Tucciarone J, Manoocheri K, Snider WG, et al.

715 Cooperative Subnetworks of Molecularly Similar Interneurons in Mouse Neocortex. Neuron.

$716 \quad 2016 ; 90(1): 86-100$.

717 23. Wall NR, De La Parra M, Sorokin JM, Taniguchi H, Huang ZJ, Callaway EM. Brain-

718 Wide Maps of Synaptic Input to Cortical Interneurons. J Neurosci. 2016;36(14):4000-9. 
719 24. Turi GF, Li WK, Chavlis S, Pandi I, O'Hare J, Priestley JB, et al. Vasoactive Intestinal

720 Polypeptide-Expressing Interneurons in the Hippocampus Support Goal-Oriented Spatial

721 Learning. Neuron. 2019;101(6):1150-65 e8.

722 25. Ferezou I, Cauli B, Hill EL, Rossier J, Hamel E, Lambolez B. 5-HT3 receptors mediate

723 serotonergic fast synaptic excitation of neocortical vasoactive intestinal peptide/cholecystokinin

724 interneurons. J Neurosci. 2002;22(17):7389-97.

725 26. Poorthuis RB, Enke L, Letzkus JJ. Cholinergic circuit modulation through differential

726 recruitment of neocortical interneuron types during behaviour. J Physiol. 2014;592(19):4155-64.

727 27. Pronneke A, Witte M, Mock M, Staiger JF. Neuromodulation Leads to a Burst-Tonic

728 Switch in a Subset of VIP Neurons in Mouse Primary Somatosensory (Barrel) Cortex. Cereb

729 Cortex. 2020;30(2):488-504.

730 28. Taniguchi H, He M, Wu P, Kim S, Paik R, Sugino K, et al. A resource of Cre driver lines

731 for genetic targeting of GABAergic neurons in cerebral cortex. Neuron. 2011;71(6):995-1013.

732 29. Kohara K, Pignatelli M, Rivest AJ, Jung HY, Kitamura T, Suh J, et al. Cell type-specific

733 genetic and optogenetic tools reveal hippocampal CA2 circuits. Nat Neurosci. 2014;17(2):269-

73479.

735 30. Haubensak W, Kunwar PS, Cai H, Ciocchi S, Wall NR, Ponnusamy R, et al. Genetic

736 dissection of an amygdala microcircuit that gates conditioned fear. Nature. 2010;468(7321):270-

7376.

738 31. Cruz-Martin A, El-Danaf RN, Osakada F, Sriram B, Dhande OS, Nguyen PL, et al. A

739 dedicated circuit links direction-selective retinal ganglion cells to the primary visual cortex.

$740 \quad$ Nature. 2014;507(7492):358-61. 
741 32. Ghosh KK, Burns LD, Cocker ED, Nimmerjahn A, Ziv Y, Gamal AE, et al. Miniaturized

742 integration of a fluorescence microscope. Nat Methods. 2011;8(10):871-8.

743 33. Liberti WA, Perkins LN, Leman DP, Gardner TJ. An open source, wireless capable

744 miniature microscope system. J Neural Eng. 2017;14(4):045001.

745 34. Allen Mouse Brain Atlas. 2004.

746 35. Aharoni D, Khakh BS, Silva AJ, Golshani P. All the light that we can see: a new era in 747 miniaturized microscopy. Nat Methods. 2019;16(1):11-3.

748 36. Comer AL, Sriram B, Yen WW, Cruz-Martin A. A Pipeline using Bilateral In Utero 749 Electroporation to Interrogate Genetic Influences on Rodent Behavior. J Vis Exp. 2020(159).

750 37. Mathis A, Mamidanna P, Cury KM, Abe T, Murthy VN, Mathis MW, et al. DeepLabCut: 751 markerless pose estimation of user-defined body parts with deep learning. Nat Neurosci.

$752 \quad 2018 ; 21(9): 1281-9$.

753 38. Comer AL, Jinadasa T, Sriram B, Phadke RA, Kretsge LN, Nguyen TPH, et al. Increased 754 expression of schizophrenia-associated gene C4 leads to hypoconnectivity of prefrontal cortex 755 and reduced social interaction. PLoS Biol. 2020;18(1):e3000604.

756 39. Giovannucci A, Friedrich J, Gunn P, Kalfon J, Brown BL, Koay SA, et al. CaImAn an 757 open source tool for scalable calcium imaging data analysis. Elife. 2019;8.

758 40. Virtanen P, Gommers R, Oliphant TE, Haberland M, Reddy T, Cournapeau D, et al. 759 SciPy 1.0: fundamental algorithms for scientific computing in Python. Nat Methods.

$760 \quad 2020 ; 17(3): 261-72$.

761 41. Li Y, Mathis A, Grewe BF, Osterhout JA, Ahanonu B, Schnitzer MJ, et al. Neuronal

762 Representation of Social Information in the Medial Amygdala of Awake Behaving Mice. Cell. $763 \quad 2017 ; 171(5): 1176-90$ e17. 
764 42. Kingsbury L, Huang S, Raam T, Ye LS, Wei D, Hu RK, et al. Cortical Representations of

765 Conspecific Sex Shape Social Behavior. Neuron. 2020;107(5):941-53 e7.

766 43. Callaway EM, Luo L. Monosynaptic Circuit Tracing with Glycoprotein-Deleted Rabies

767 Viruses. J Neurosci. 2015;35(24):8979-85.

768 44. Di Martino A, Ross K, Uddin LQ, Sklar AB, Castellanos FX, Milham MP. Functional

769 brain correlates of social and nonsocial processes in autism spectrum disorders: an activation

770 likelihood estimation meta-analysis. Biol Psychiatry. 2009;65(1):63-74.

771 45. Fuchs PN, Peng YB, Boyette-Davis JA, Uhelski ML. The anterior cingulate cortex and

772 pain processing. Front Integr Neurosci. 2014;8:35.

773 46. Galarreta M, Hestrin S. A network of fast-spiking cells in the neocortex connected by

774 electrical synapses. Nature. 1999;402(6757):72-5.

775 47. Shibata H. Efferent projections from the anterior thalamic nuclei to the cingulate cortex

776 in the rat. J Comp Neurol. 1993;330(4):533-42.

777 48. Shibata H, Kondo S, Naito J. Organization of retrosplenial cortical projections to the

778 anterior cingulate, motor, and prefrontal cortices in the rat. Neurosci Res. 2004;49(1):1-11.

779 49. Shibata H, Naito J. Organization of anterior cingulate and frontal cortical projections to

780 the retrosplenial cortex in the rat. J Comp Neurol. 2008;506(1):30-45.

781 50. Shibata H, Naito J. Organization of anterior cingulate and frontal cortical projections to

782 the anterior and laterodorsal thalamic nuclei in the rat. Brain Res. 2005;1059(1):93-103.

783 51. Aggleton JP, Pralus A, Nelson AJ, Hornberger M. Thalamic pathology and memory loss

784 in early Alzheimer's disease: moving the focus from the medial temporal lobe to Papez circuit.

785 Brain. 2016;139(Pt 7):1877-90. 
786 52. Van der Werf YD, Witter MP, Uylings HB, Jolles J. Neuropsychology of infarctions in

787 the thalamus: a review. Neuropsychologia. 2000;38(5):613-27.

788 53. Perry BAL, Mitchell AS. Considering the Evidence for Anterior and Laterodorsal

789 Thalamic Nuclei as Higher Order Relays to Cortex. Front Mol Neurosci. 2019;12:167.

790 54. Tanimizu T, Kenney JW, Okano E, Kadoma K, Frankland PW, Kida S. Functional

791 Connectivity of Multiple Brain Regions Required for the Consolidation of Social Recognition

792 Memory. J Neurosci. 2017;37(15):4103-16.

793 55. Krabbe S, Paradiso E, d'Aquin S, Bitterman Y, Courtin J, Xu C, et al. Adaptive

794 disinhibitory gating by VIP interneurons permits associative learning. Nat Neurosci.

$795 \quad 2019 ; 22(11): 1834-43$.

796 56. Deng YZ, Lu YC, Wu WW, Cheng L, Zan GY, Chai JR, et al. Anteromedial thalamic

797 nucleus to anterior cingulate cortex inputs modulate histaminergic itch sensation.

798 Neuropharmacology. 2020;168:108028.

799 57. Alexander AS, Carstensen LC, Hinman JR, Raudies F, Chapman GW, Hasselmo ME.

800 Egocentric boundary vector tuning of the retrosplenial cortex. Sci Adv. 2020;6(8):eaaz2322.

801 58. Fischer LF, Mojica Soto-Albors R, Buck F, Harnett MT. Representation of visual

802 landmarks in retrosplenial cortex. Elife. 2020;9.

803 59. Vann SD, Aggleton JP, Maguire EA. What does the retrosplenial cortex do? Nat Rev

804 Neurosci. 2009;10(11):792-802.

805 60. Michael J. Proulx OST, Amanda Taylor Aiken and Alexandra A. de Sousa. Where am I?

806 Who am I? The Relation Between Spatial Cognition, Social Cognition and Individual

807 Differences in the Built Environment. Front Psychol. 2016;11. 
808 61. Gehring WJ, Knight RT. Prefrontal-cingulate interactions in action monitoring. Nat

809 Neurosci. 2000;3(5):516-20.

810 62. Paxinos F. The Mouse Brain in Stereotaxic Coordinates, 4th Edition. 2012

811 63. van Heukelum S, Mars RB, Guthrie M, Buitelaar JK, Beckmann CF, Tiesinga PHE, et al.

812 Where is Cingulate Cortex? A Cross-Species View. Trends Neurosci. 2020;43(5):285-99.

813 64. Fillinger C, Yalcin I, Barrot M, Veinante P. Afferents to anterior cingulate areas 24a and

$81424 b$ and midcingulate areas 24a' and 24b' in the mouse. Brain Struct Funct. 2017;222(3):1509-

81532.

816 65. Hoover WB, Vertes RP. Anatomical analysis of afferent projections to the medial

817 prefrontal cortex in the rat. Brain Struct Funct. 2007;212(2):149-79.

818 66. Tasic B, Menon V, Nguyen TN, Kim TK, Jarsky T, Yao Z, et al. Adult mouse cortical

819 cell taxonomy revealed by single cell transcriptomics. Nat Neurosci. 2016;19(2):335-46.

820 67. Tasic B, Yao Z, Graybuck LT, Smith KA, Nguyen TN, Bertagnolli D, et al. Shared and

821 distinct transcriptomic cell types across neocortical areas. Nature. 2018;563(7729):72-8.

822 68. Zhang S, Xu M, Kamigaki T, Hoang Do JP, Chang WC, Jenvay S, et al. Selective

823 attention. Long-range and local circuits for top-down modulation of visual cortex processing.

824 Science. 2014;345(6197):660-5.

825 69. Letzkus JJ, Wolff SB, Luthi A. Disinhibition, a Circuit Mechanism for Associative

826 Learning and Memory. Neuron. 2015;88(2):264-76.

827 70. Berridge CW, Waterhouse BD. The locus coeruleus-noradrenergic system: modulation of

828 behavioral state and state-dependent cognitive processes. Brain Res Brain Res Rev.

$829 \quad 2003 ; 42(1): 33-84$. 
830 71. Aston-Jones G, Rajkowski J, Cohen J. Role of locus coeruleus in attention and behavioral

831 flexibility. Biol Psychiatry. 1999;46(9):1309-20.

832 72. Uematsu A, Tan BZ, Johansen JP. Projection specificity in heterogeneous locus coeruleus

833 cell populations: implications for learning and memory. Learn Mem. 2015;22(9):444-51.

834 73. Sara SJ, Bouret S. Orienting and reorienting: the locus coeruleus mediates cognition

835 through arousal. Neuron. 2012;76(1):130-41.

836 74. Gompf HS, Mathai C, Fuller PM, Wood DA, Pedersen NP, Saper CB, et al. Locus

837 ceruleus and anterior cingulate cortex sustain wakefulness in a novel environment. J Neurosci.

$838 \quad 2010 ; 30(43): 14543-51$.

839 75. Kawaguchi Y, Shindou T. Noradrenergic excitation and inhibition of GABAergic cell

840 types in rat frontal cortex. J Neurosci. 1998;18(17):6963-76.

841 76. McCormick DA, Prince DA. Noradrenergic modulation of firing pattern in guinea pig

842 and cat thalamic neurons, in vitro. J Neurophysiol. 1988;59(3):978-96.

843 77. Kampa BM, Letzkus JJ, Stuart GJ. Cortical feed-forward networks for binding different

844 streams of sensory information. Nat Neurosci. 2006;9(12):1472-3.

845 78. Gehring WJ, Knight RT. Lateral prefrontal damage affects processing selection but not

846 attention switching. Brain Res Cogn Brain Res. 2002;13(2):267-79.

847 79. Pronneke A, Scheuer B, Wagener RJ, Mock M, Witte M, Staiger JF. Characterizing VIP

848 Neurons in the Barrel Cortex of VIPcre/tdTomato Mice Reveals Layer-Specific Differences.

849 Cereb Cortex. 2015;25(12):4854-68.

850

851 FIGURE LEGENDS 
853 Figure 1. Distinct VIP ${ }^{A C C}$ interneurons preferentially encode anxiogenic or anxiolytic the GRIN lens and GCaMP6f expression. Blue: DAPI, green: VIPACC expressing GCaMP6f. Dotted white overlay, brain regions. Yellow arrowheads, GRIN lens location in the ACC. Left: left hemisphere. Right: zoomed image of yellow boxed region. Scale bar $=1 \mathrm{~mm}$ (left panel) or

$858400 \mu \mathrm{m}$ (right panel). (C) VIP ACC expressing GCaMP6f (white) in vivo while mouse navigates the open and closed arms of the EZM. Pink circles: open-selective, green circles: closed-selective.

860 Neutral cells are not circled. Scale bar $=100 \mu \mathrm{m}$. (D) ROC curves demonstrating how well cells 861 encode behavioral states for open-selective (pink, auROC $=0.75)$, closed-selective (green, auROC $862=0.66$ ), and neutral (gray, auROC $=0.51) \mathrm{VIP}^{\mathrm{ACC}} .(\mathbf{E}) \mathrm{Ca}^{2+}$ transients: closed-selective (top), 863 open-selective (bottom). Shaded areas indicate the location of the mouse: open (pink) or closed 864 (green) arms. Scale bar $=25 \mathrm{~s}$ and 2 SD. (F) $21 \%$ of VIP ${ }^{A C C}$ were classified as open-selective, $86511 \%$ as closed-selective, and $68 \%$ as neutral. (G-I) $\mathrm{Ca}^{2+}$ activity of selective VIP ${ }^{\mathrm{ACC}}$ per mouse. (G) Closed-selective. Closed vs. open. ${ }^{* *} p=0.0016$. (H) Open-selective. Open vs. closed. $* * * p=0.0002$. (I) Neutral cells in the EZM. Closed vs. open. $p=0.5239$. (J) Movement of a mouse from an open to a closed arm (left) or a closed to an open arm (right) of the EZM. (K-L) Activity 869 of closed-selective cells from $10 \mathrm{~s}$ prior to $10 \mathrm{~s}$ after entering either a closed (K) or an open (L) 870 arm. (M-N) Activity of open-selective from $10 \mathrm{~s}$ prior to $10 \mathrm{~s}$ after entering either an open (M) or 871 closed (N) arm. (O-R) Average of selective-cell $\mathrm{Ca}^{2+}$ activity per mouse. (O) Closed-selective 872 entering closed arm. -5 vs. +5 s. $* * p=0.0046$. (P) Closed-selective entering open arm. -5 vs. +5 s. $873 * * p=0.0056$. (Q) Open-selective entering open arm. -5 vs. +5 s. $* * * p=0.0004$. (R) Open-selective 874 entering closed arm. -5 vs. +5 s. ${ }^{*} p=0.0015 . \mathrm{N}=6$ mice, $\mathrm{n}=345$ cells. All statistics performed 875 with Paired t-test. For all figures, $\mathrm{Ca}^{2+}$ activity refers to the area under the curve (integral df/f). All 
ROC curves, traces, and images are representative. Each replicate in G-I and O-R represents one mouse. C: closed arm, O: open arm, Closed-sel: closed selective, Open-sel: open-selective, df/f: Deltaf/f.

Figure 2. Distinct subpopulations of VIPACC encode social and non-social stimuli. (A)

881 Behavioral paradigm. Left: Sociability (Day 1). Right: Social Novelty (Days 2/3). Littermate zone

882 (purple). Empty cup zone (pink). Novel mouse zone (orange). Neutral zone (white). (B)

883 Sociability. Interaction Time (\%). Cup vs. littermate. ${ }^{*} p=0.0199$. (C) Social Novelty. Interaction

884 Time (\%). Littermate vs. novel mouse. ${ }^{*} p=0.0141$. (D) Images of VIPACC expressing GCaMP6f

885 (white) in vivo during Sociability (left) or Social Novelty (right). Pink circles: cup-selective,

886 purple circles: littermate-selective, orange circles: novel mouse-selective. Neutral cells are not

887 circled. Scale bar $=100 \mu \mathrm{m}$. (E) ROC curves for littermate-selective (purple, auROC $=0.72$ ),

888 novel-mouse-selective (orange, auROC $=0.67$ ), cup-selective (pink, auROC $=0.69$ ), and neutral

889 (gray, auROC $=0.51)$ VIP ${ }^{\mathrm{ACC}}$. (F) $\mathrm{Ca}^{2+}$ transients: littermate-selective (top), cup-selective

890 (middle), neutral cell (bottom). Shaded areas indicate location of mouse: littermate (purple), cup

891 (pink), neutral (white) zones. Scale bars $=25 \mathrm{~s}$ and 1 SD. (G) In Sociability, 17\% of VIPACC were $^{\text {AC }}$

892 classified as cup-selective, $15 \%$ as littermate-selective, $2 \%$ as selective for both cup and littermate,

893 and $66 \%$ as neutral. In Social Novelty, VIPACC were classified as littermate-selective (11\% Day 2 ,

894 20\% Day 3), novel-mouse-selective (24\% Day 2, 12\% Day 3), selective for both littermate and

895 novel (1\% Day 2, 0\% Day 3), and neutral (64\% Day 2, 68\% Day 3). (H-K) Average $\mathrm{Ca}^{2+}$ activity

896 of selective cells per mouse. (H) Littermate-selective. Sociability. Littermate vs. cup. ${ }^{*} p=0.0132$.

897 (I) Empty cup-selective. Sociability. Cup vs. littermate. ${ }^{* *} p=0.0017$. (J) Littermate-selective.

898 Social Novelty. Littermate vs. novel mouse. ${ }^{* * * *} p<0.0001$. (K) Novel-mouse-selective. Social 
Novelty. Littermate vs. novel mouse. ${ }^{* * *} p<0.0001 . \mathrm{N}=6$ mice, $\mathrm{n}=310$ cells for Sociability, $\mathrm{n}=$ 350 cells for Social Novelty Day 2, and $n=232$ cells for Day 3. All ROC curves, traces, and

901 images are representative. Each replicate in B-C and H-K represents one mouse. All statistics 902 performed with Paired t-test. Lit: littermate, Nov: novel mouse, Lit-sel: littermate-selective cell, 903 Cup-sel: cup-selective cell. Social nov: Social Novelty, df/f: Deltaf/f. curves of open- (pink solid, auROC $=0.86)$, closed- (green, auROC $=0.70)$, littermate- (purple, auROC $=0.74)$, empty cup- (pink dotted, auROC $=0.70)$, and novel-mouse-selective (orange, auROC $=0.79)$ super cells. $(\mathbf{B}-\mathbf{D}) \mathrm{Ca}^{2+}$ transients of each type of super cell for the EZM (B) and social interaction tasks $(\mathbf{C}-\mathbf{D})$. Scale bars $=10 \mathrm{~s}$ and $1 \mathrm{SD}$. Shaded areas represent location of the mouse. Closed arm (B, green), open arm (B, pink), littermate (C-D, purple), empty cup (C, pink),

911 novel mouse (D, orange) or neutral (C-D, white) zones. $\mathrm{Ca}^{2+}$ signals overlayed. (E-J) auROC of

912 all cells (All), selective cells, and super cells (Super) per mouse in the EZM (E-F) and social 913 interaction tasks (G-J). In each task, selective cells had higher auROC than the whole population

914 and super cells had higher auROC values than selective cells. (E-F) This pattern was consistent in

915 the EZM for both closed- (E, All vs. closed: $* * * * p<0.0001$. All vs. super: ${ }^{* * * *} p<0.0001$. Closed 916 vs. super: $\left.{ }^{* * * *} p<0.0001\right)$ and open-selective cells (F, All vs. open: ${ }^{* * *} p=0.0009$. All vs. super:

$917{ }^{* *} p=0.0047$. Open vs. super: $\left.{ }^{*} p=0.0370\right) .(\mathbf{G}-\mathbf{H})$ In Sociability, this pattern was consistent for

918 littermate- (G, All vs. littermate: ${ }^{* *} p=0.0022$. All vs. super: ${ }^{* *} p=0.0011$. Littermate vs. super: $\left.919{ }^{*} p=0.0326\right)$ and cup-selective (H, All vs. cup: ${ }^{* *} p=0.0026$. All vs. super: ${ }^{* *} p=0.0003$. Cup vs. 920 super: $\left.{ }^{* *} p=0.0010\right)$. (I-J) In Social Novelty, this pattern was consistent for littermate- (I, All vs. 921 littermate: ${ }^{* * *} p=0.0005$. All vs. super: ${ }^{* * *} p=0.0008$. Littermate vs. super: ${ }^{* *} p=0.0073$.) and 
922

923

924

925

926

927

928

929

930

931

932

933

934

935

936

937

938

939

940

941

942

943

944

novel-mouse-selective (J, All vs. novel: $* * p=0.0059$. All vs. super: $* * * * p<0.0001$. Novel vs. super: $* * * p=0.0007) . * * * * p<0.0001 . \quad \mathrm{N}=6$ mice, $\mathrm{n}=345$ cells for $\mathrm{EZM}, \mathrm{n}=310$ cells for Sociability, $n=350$ cells for Social Novelty Day $2, n=232$ cells and Day 3 . All ROC curves and traces are representative. Each replicate in E-J represents one mouse. All statistics performed with Repeated measures ANOVA with Tukey’s post test. Lit: littermate, Soc: social.

\section{Figure 4. VIP ${ }^{\mathrm{ACC}}$ subpopulations are non-overlapping and are recruited during distinct}

behaviors. (A-B) VIP ${ }^{\mathrm{ACC}}$ were registered across tasks. Purple circles: registered cells monitored during both EZM (A) and Sociability (B). (A-B) Left: Scale bar $=100 \mu \mathrm{m}$. Right: zoomed versions of yellow outlined regions in left panels. Scale bar $=40 \mu \mathrm{m}$. (C) Venn diagram demonstrating cell selectivity in the EZM and Sociability. Each number indicates what percentage of the registered cells belong to each selectivity category. There is a gap between open and closed because these classifications are mutually exclusive - by definition a cell cannot be selective for both. (D) Percentage of cells selective for one, two, or three stimuli. Dotted line, one phase decay, half-life of 0.81. (E) Image of starter VIPACC expressing both EGFP (pseudo colored magenta, also expressing TVA and $\Delta \mathrm{G}$ ) and mCherry (pseudo colored yellow, infected by RVdG), and retrogradely-labeled input cells only expressing mCherry. Coronal section. Left: overlay of both fluorophores and DAPI (cyan). Scale bar $=50 \mu \mathrm{m}$. Right: zoomed in superficial layers showing overlap (starter cells, white arrows). Scale bar $=15 \mu \mathrm{m}$. (F) Images of retrogradely-labeled input neurons (white) in the orbitofrontal (Orb) cortex (Ctx), anterior thalamic nuclei (Am Thal or AM), retrosplenial (RS) cortex, and diagonal band of Broca (Diag Band, part of the medial septal complex, MSC). Left panels: left hemisphere. Scale bar $=1.5 \mathrm{~mm}$. Right panels: zoomed views of the retrogradely-labeled brain regions (yellow arrowheads in left panel). Scale bar $=100 \mu \mathrm{m}$. 
945 (G-J) Regions that are the most highly connected to VIP ${ }^{\text {ACC }}$. Rabies trans-synaptic mapping revealed that the prefrontal Ctx (PFC), primary and association Ctx (Prim/asso), thalamic regions,

947 and MSC are highly connected to VIPACC. (H-J) Highly connected areas (PFC, H, Prim/asso, I, 948 thalamic, J) divided into subregions. Error bars represent SEM. N $=6$ mice for $\mathrm{Ca}^{2+}$ imaging, $\mathrm{n}=$ 949345 cells for EZM, $n=310$ cells for Sociability. For tracing experiments, $N=3$ mice with $n=691$ 950 starter cells and 10107 input cells. All images are representative. Pref Stim: preferred stimuli, 951 Hypo: hypothalamus, Amyg: amygdala, cl: contralateral, PrL: prelimbic Ctx, VO: ventral orbital 952 Ctx, MO: medial orbital Ctx, LO: lateral orbital Ctx, RSG: restrosplenial granular Ctx, RSD: 953 retrosplenial dysgranular Ctx, V2MM: secondary visual Ctx mediomedial area, S1BF: primary 954 somatosensory Ctx barrel field, MPtA: medial parietal association Ctx, S1: primary somatosensory 955 Ctx, V1: primary visual Ctx, LPMR: lateral posterior thalamic nucleus medio rostral part, PF: 956 parafascicular thalamic nucleus, MDL: mediodorsal thalamic nucleus lateral part, LDVL: 957 laterodorsal thalamic nucleus ventrolateral part, CL: centrolateral thalamic nucleus, VA: ventral 958 anterior thalamic nucleus, AD: anterodorsal thalamic nucleus.

\section{AVAILABILITY OF DATA AND MATERIALS}

962 Data Availability: All relevant data are within the paper, and underlying data are available at 963 https://github.com/CruzMartinLab. Custom-written routines for behavioral tracking, calcium 964 imaging analysis, and miniscope models and tools are available at 965 https://github.com/CruzMartinLab. For further information, please contact the corresponding 966 author. 
Figure 1

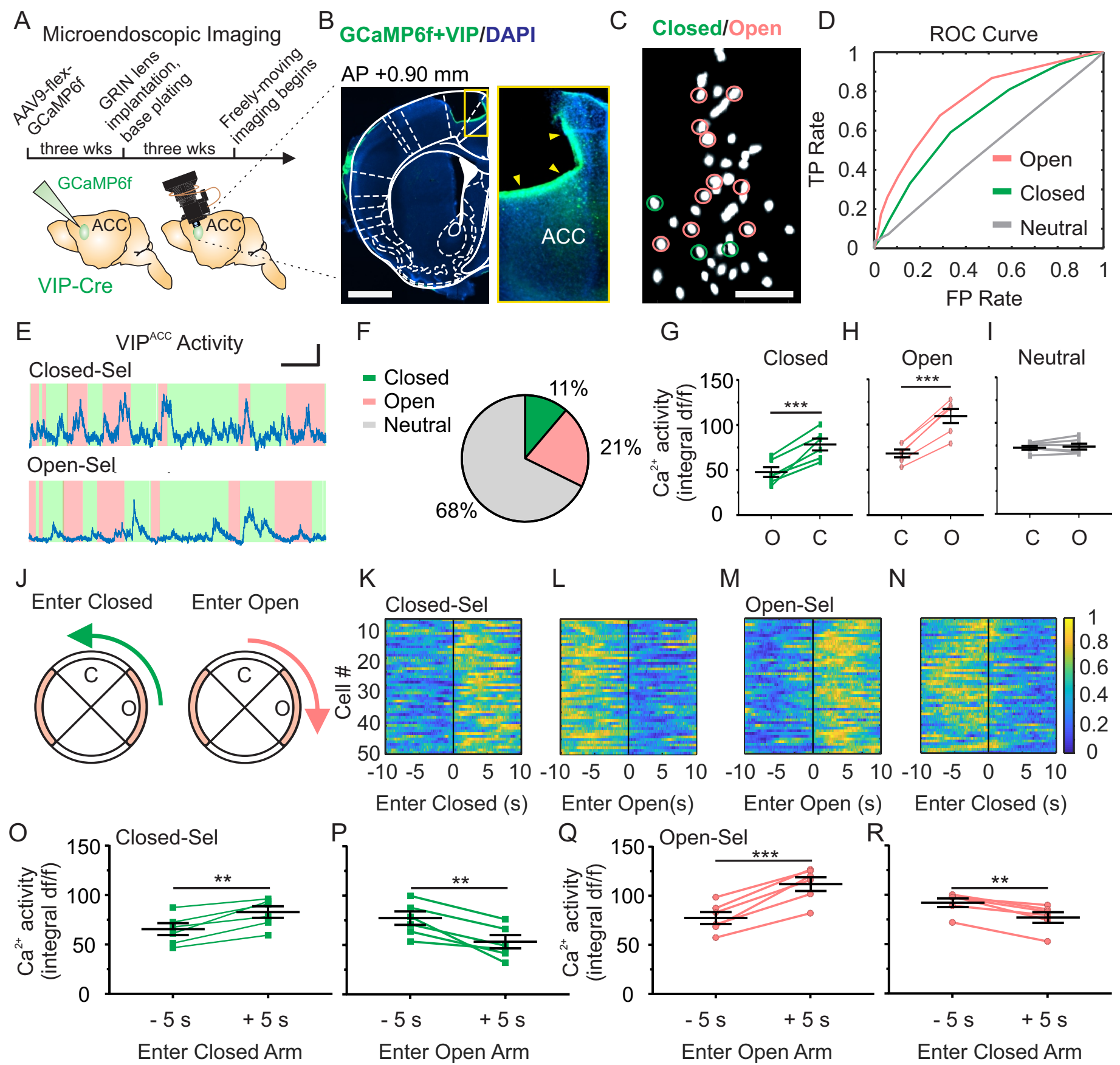


Figure 2

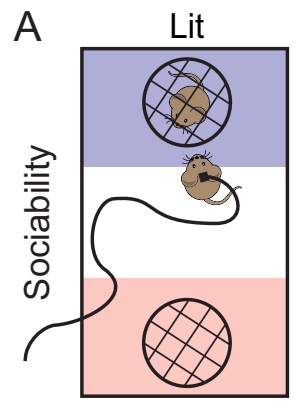

Cup

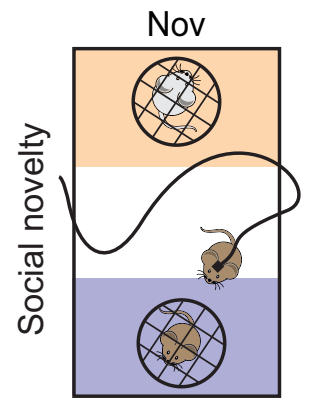

Lit
B

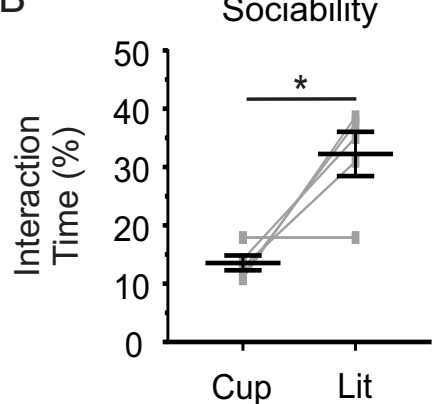

C Social novelty

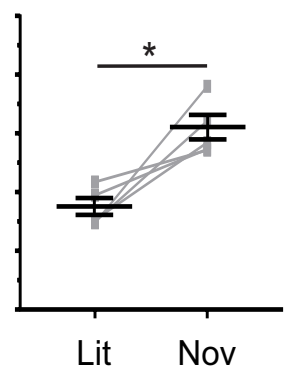

D Sociability

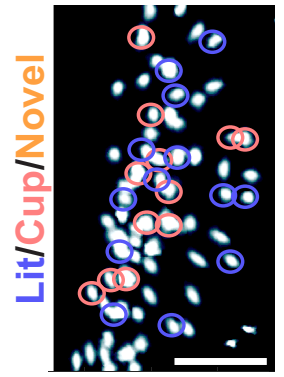

Social novelty

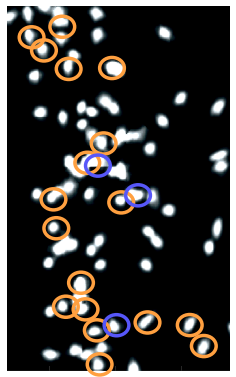

E

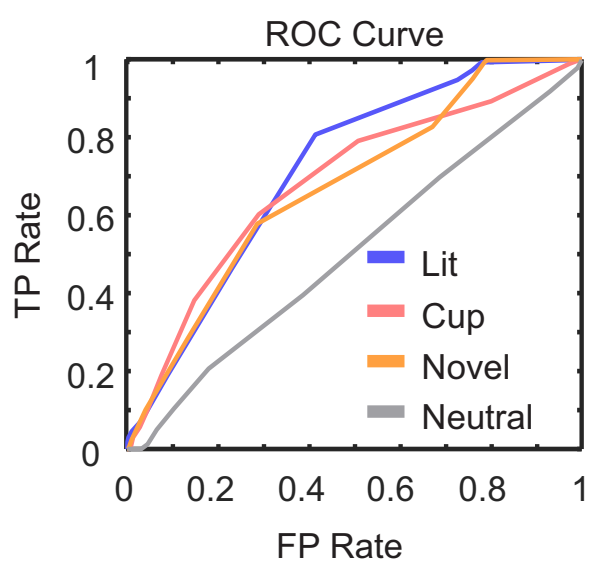

$\mathrm{F}$

VIP ${ }^{A C C}$ Activity

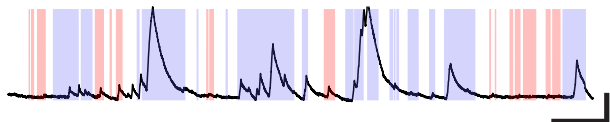

Cup-Sel (Sociability)

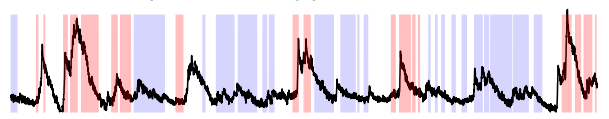

Neutral (Sociability)

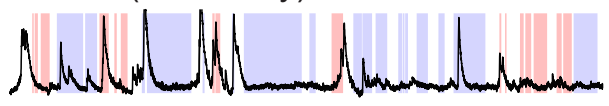

$\mathrm{H}$

$$
\text { Lit (Sociability) }
$$
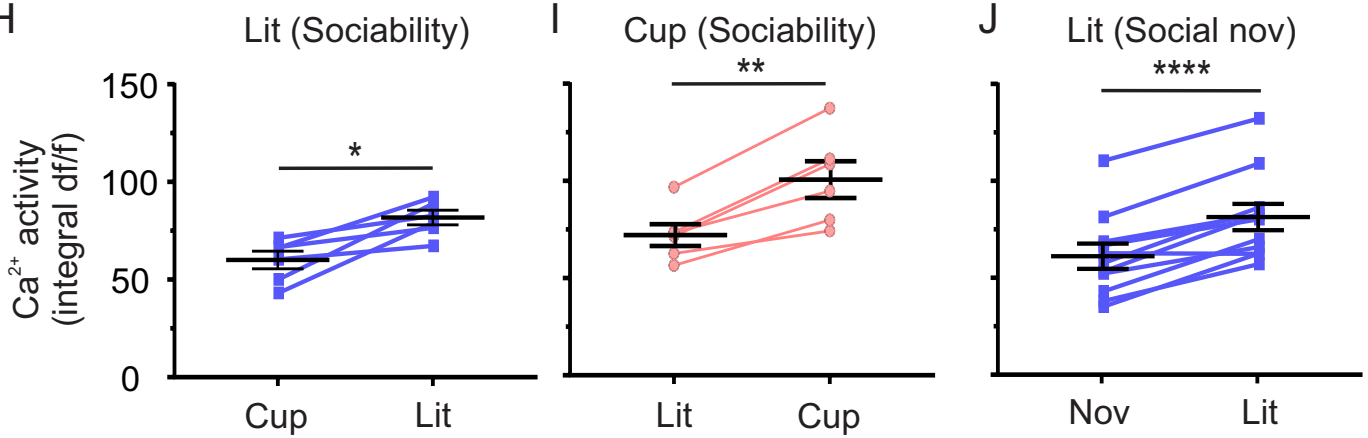
G - Lit - Neutral - Lit/Cup
- Cup - Novel - Lit/Novel
Social novelty

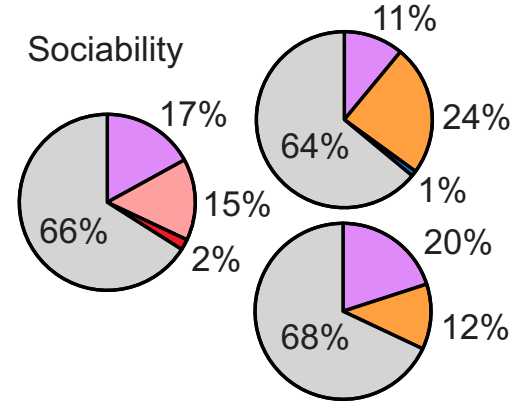

K Novel (Social nov)

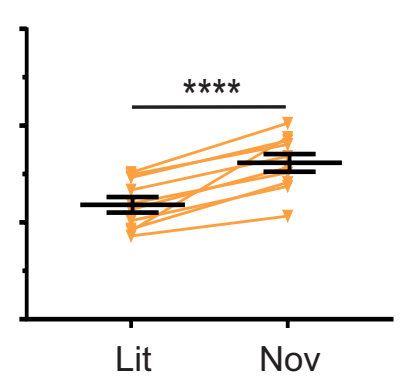


Figure 3
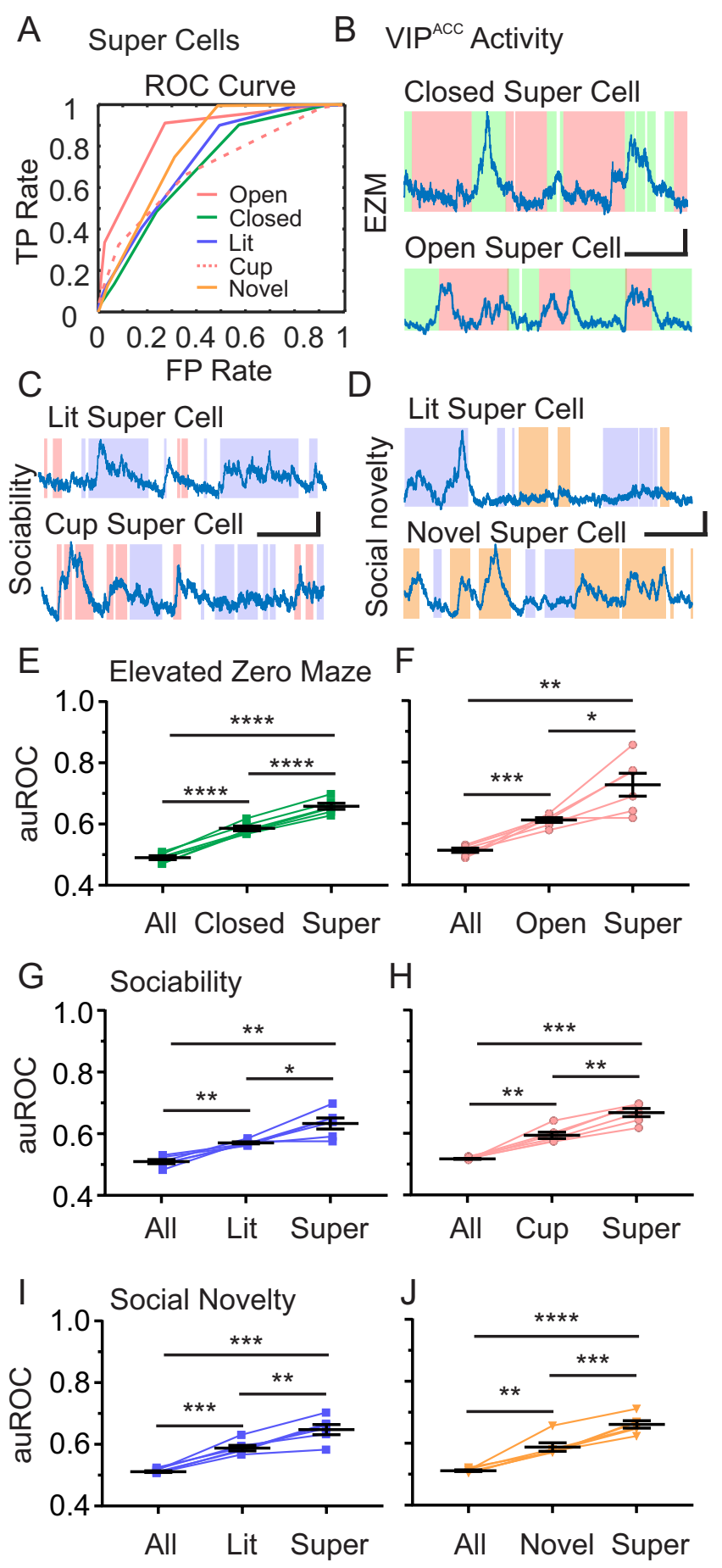
Figure 4

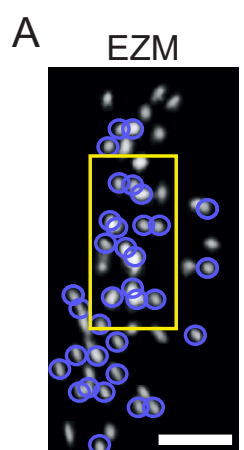

E

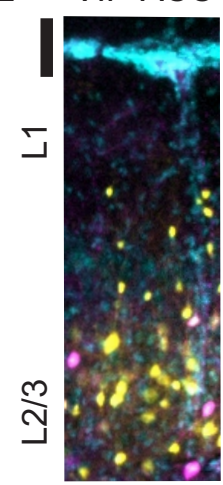

$\mathrm{H}$

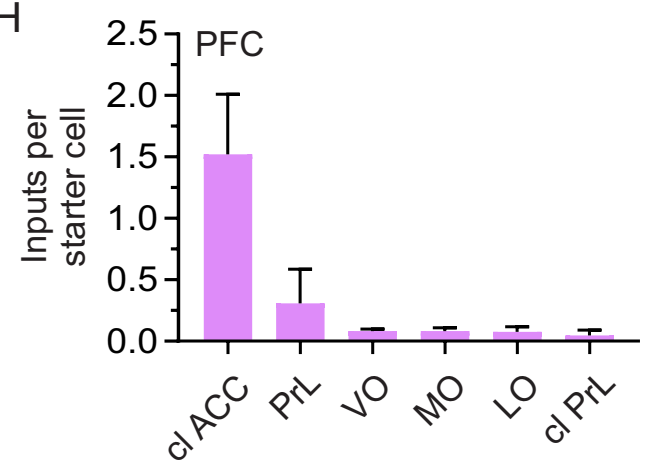

Sociability

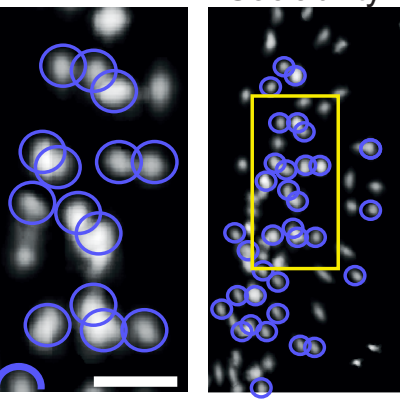

F Inputs
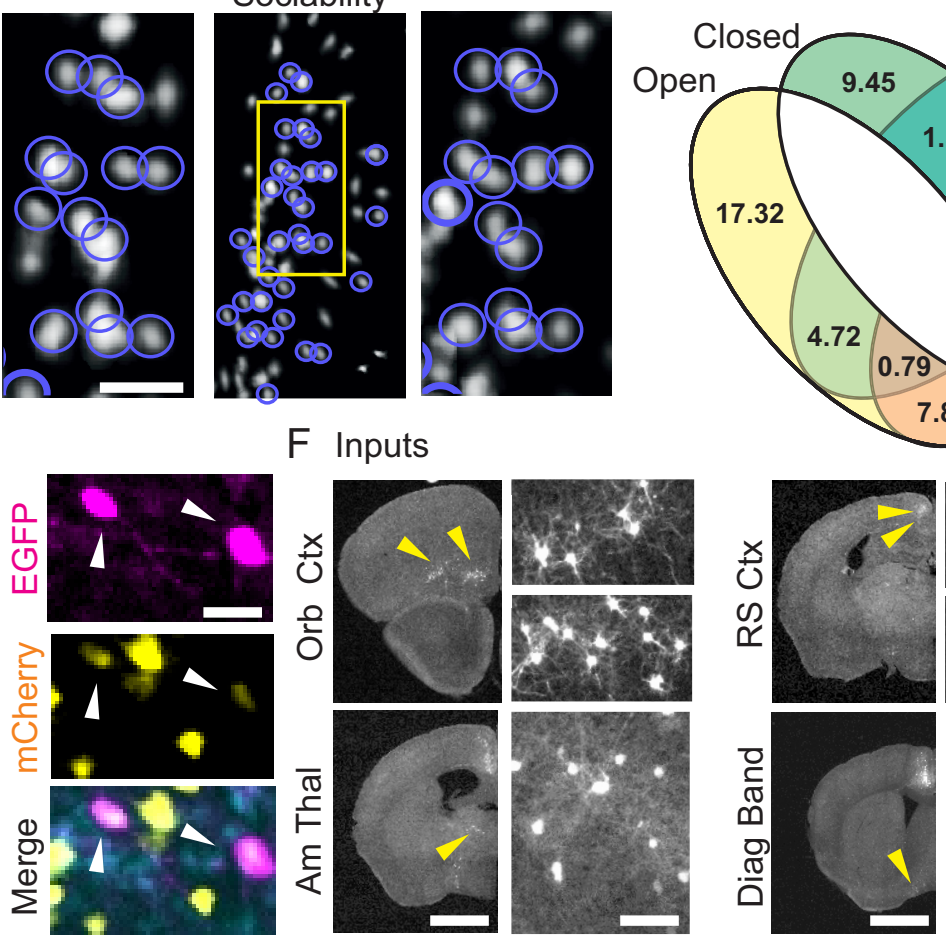

C
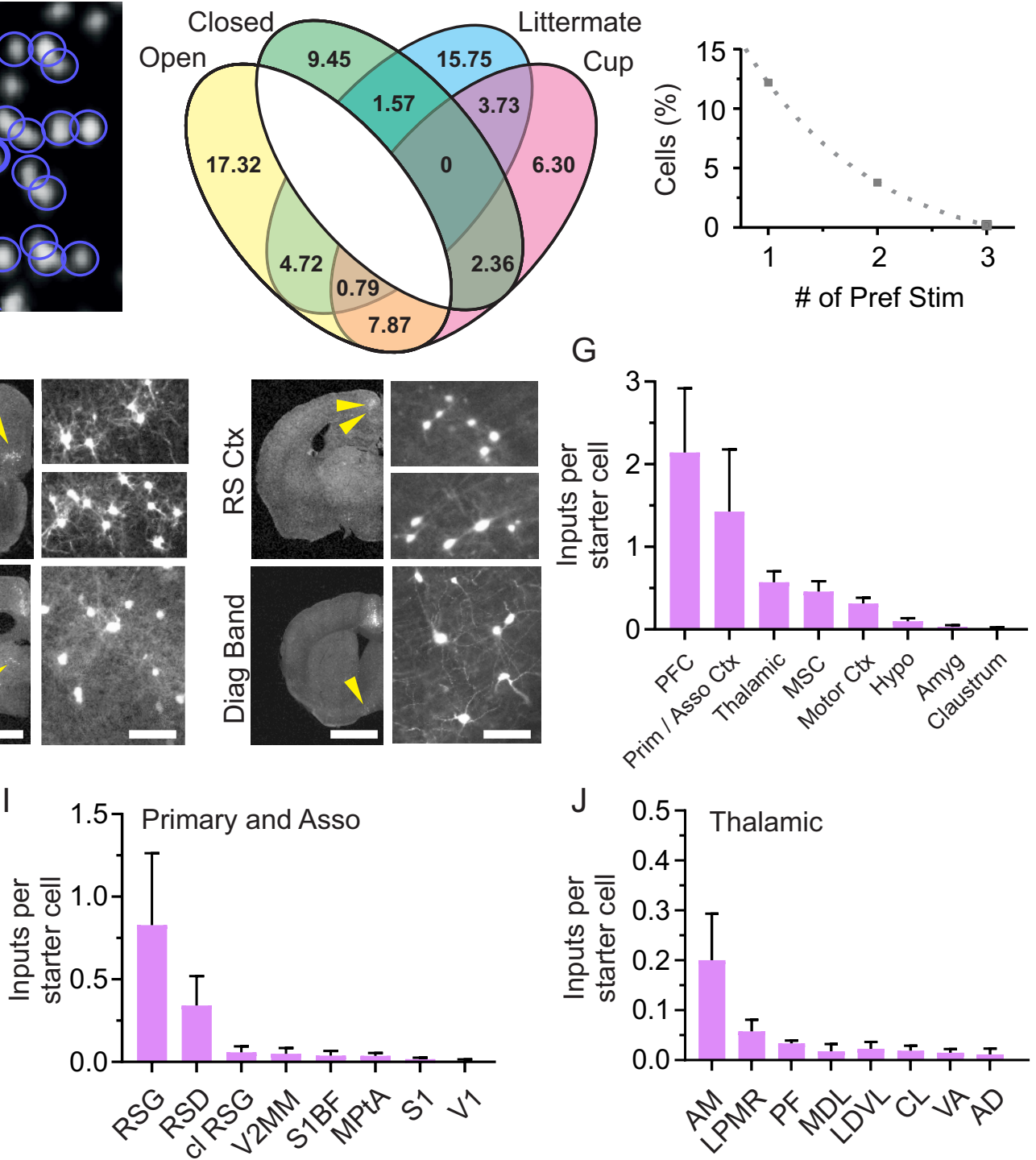

J

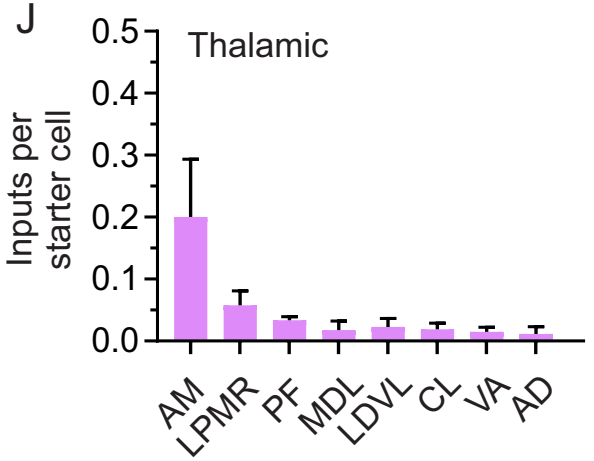

